\title{
Should I stay or should I go? Conceptual underpinnings of goal-directed actions
}

\author{
Giovanni Mirabella ${ }^{1,2}$ * \\ ${ }^{1}$ Istituto Neurologico Mediterraneo, IRCCS Neuromed, Pozzilli, Italy \\ 2 Department of Physiology and Pharmacology 'V. Erspamer,' La Sapienza University, Rome, Italy
}

\section{Edited by:}

Manuel Casanova, University of

Louisville, USA

\section{Reviewed by:}

Satoshi Tsujimoto, Kobe University, Japan

Mikhail Lebedev, Duke University, USA

\section{*Correspondence:}

Giovanni Mirabella, Istituto Neurologico Mediterraneo, IRCCS Neuromed, via Atinense 18, 86077 Pozzilli, Italy

e-mail: giovanni.mirabella@uniroma1.it
All actions, even the simplest like moving an arm to grasp a pen, are associated with energy costs. Thus all mobile organisms possess the ability to evaluate resources and select those behaviors that are most likely to lead to the greatest accrual of valuable items (reward) in the near or, especially in the case of humans, distant future. The evaluation process is performed at all possible stages of the series of decisions that lead to the building of a goal-directed action or to its suppression. This is because all animals have a limited amount of energy and resources; to survive and be able to reproduce they have to minimize the costs and maximize the outcomes of their actions. These computations are at the root of behavioral flexibility. Two executive functions play a major role in generating flexible behaviors: (i) the ability to predict future outcomes of goal-directed actions; and (ii) the ability to cancel them when they are unlikely to accomplish valuable results. These two processes operate continuously during the entire course of a movement: during its genesis, its planning and even its execution, so that the motor output can be modulated or suppressed at any time before its execution. In this review, functional interactions of the extended neural network subserving generation and inhibition of goal-directed movements will be outlined, leading to the intriguing hypothesis that the performance of actions and their suppression are not specified by independent sets of brain regions. Rather, it will be proposed that acting and stopping are functions emerging from specific interactions between largely overlapping brain regions, whose activity is intimately linked (directly or indirectly) to the evaluations of pros and cons of an action. Such mechanism would allow the brain to perform as a highly efficient and flexible system, as different functions could be computed exploiting the same components operating in different configurations.

Keywords: decision-making, reward, voluntary motor control, behavioral flexibility, countermanding task, reaching arm movements

\section{INTRODUCTION}

Our survival depends on the ability to gather, parse and evaluate the stream of constantly changing environmental stimuli and to flexibly adapt our behavioral responses according to the context in which we are embedded. This is because all animals operate with limited resources, and thus the way they value their internal states, sensory experience, and behavioral output influences directly how they will invest their time and energy (Rangel et al., 2008). The bottom line is that the opportunity of executing any action needs to be continuously evaluated in order to minimize its costs and to maximize its payoffs. In fact, the value associated with a certain stimulus is not an intrinsic property of the stimulus, but can change as a function of the internal states of the agent at the time the stimulus is encountered and as a function of agent's previous experience with that stimulus. For instance, for a thirsty gazelle the water of a pond might represent a highly valuable stimulus, unless it perceives the presence of hungry lions.

Central to this process are two executive functions: (i) the ability to predict the future outcomes of a given action; and (ii) the ability to suppress inappropriate, i.e., not sufficiently valuable, actions. Importantly, these two executive functions operate not only during the genesis of an action, but also during the planning of an already selected action. In fact, during the temporal gap between the time when an action has been chosen and the moment when the motor output is going to be generated, the context might have changed, altering the computed value of the action and thus requiring a radical change of the planned motor strategy. For instance, the sight of a tasty cake is likely to drive a child to plunge his fingers into the cream, but if, when he is about to act, he suddenly feels he is observed by his parents, he will refrain from executing the planned movement. In this instance, the fear of being punished has overcome the potential reward of a sweet food, causing the suppression of the pending action.

Conceptually, a goal-directed action can be modeled as multistep decision process to which several brain regions contribute (see Figure 1; Table 1). The different stages leading up to the execution or the inhibition of an action are described in the next sections. The model I propose has been inspired by that suggested by Haggard (2008). However, there are two key differences between the former and the latter. First of all, the new model does not necessarily subserve human volition (see paragraph 'Concluding Thoughts'). Secondly, in this new 


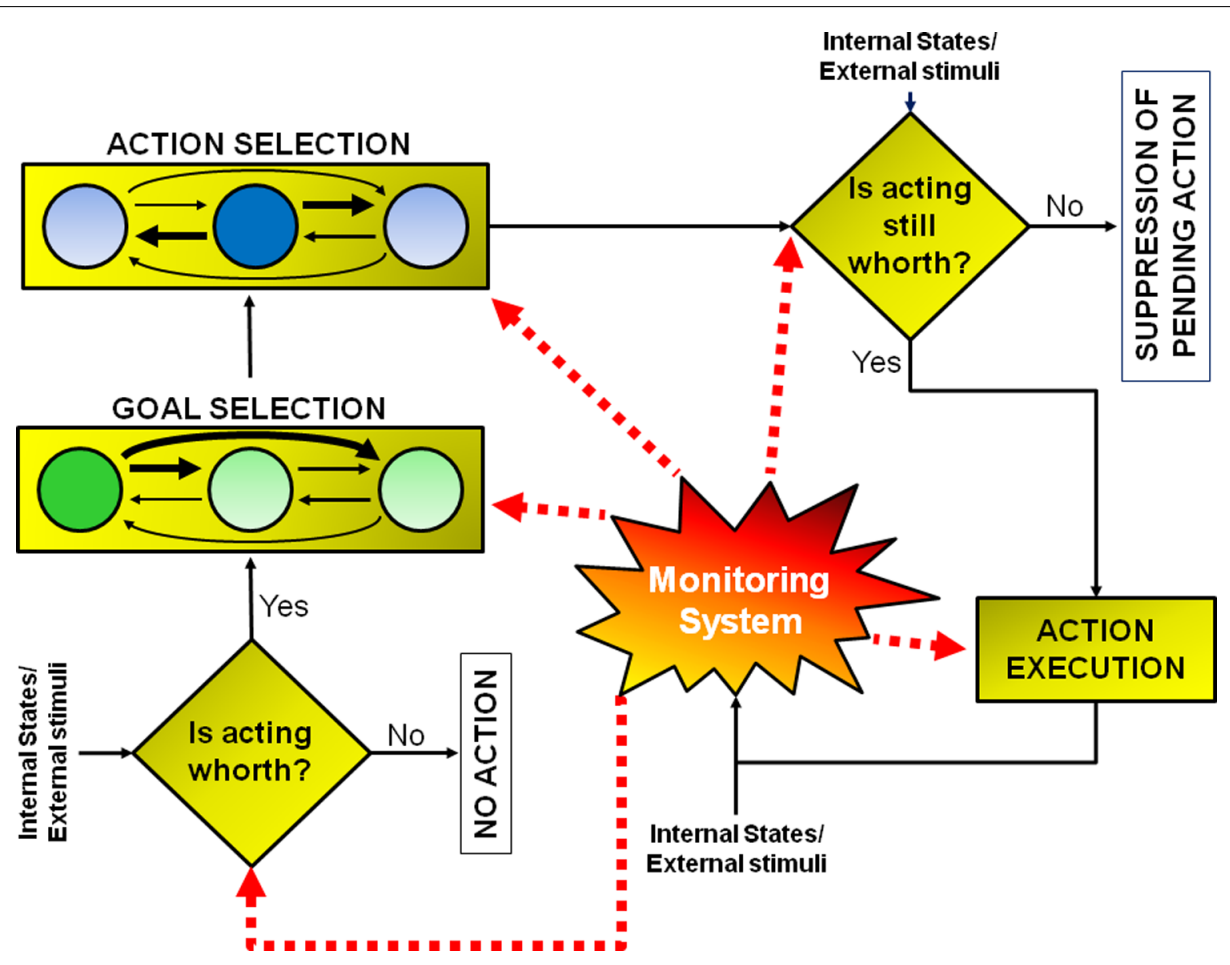

FIGURE 1 | Model of goal-directed actions. The model consists of a set of a multi-step decision processes leading either to the execution or to the inhibition of an action, according to the evaluation of its pros and cons. This model does not have a strictly serial or parallel architecture. Some processes must occur before others (e.g., the early "should-I-stay-or-should-I-go" decision aimed at evaluating whether acting is worthwhile must occur before goal or action selection), but other processes occur in parallel (e.g., the monitoring system, whose role is to compute predictions about future reward and to measure discrepancies between expected and actual outcomes, is active during all the steps). See text for further details. model, each stage of the process can be influenced by a component named monitoring system, according to the results of the outcomes of previous decisions (see paragraph 'The Monitoring System'). As a consequence the model I propose does not work strictly in a serial fashion, but both serially and in parallel.

Before proceeding, I want to remark that I will mostly deal with upper limb action control, leaving aside saccadic eye movement control. This is because the saccadic system has a different functional organization from that controlling arm and hand movements (e.g., see Churchland and Shenoy, 2007; Schall and Godlove, 2012). For instance, a number of studies have provided evidence that the inhibitory controls of eye and hand movements are independent (e.g., Logan and Irwin, 2000; Boucher et al., 2007; Sumner et al., 2007; Mirabella et al., 2011). Interestingly, Mirabella et al. (2009) and Stevenson et al. (2009) studied the effect of introducing a temporal gap between the end of the fixation and target presentation on the reaction times (RTs) and on the speed of inhibition of reaching movements and saccades, respectively. They found contrasting results: in the former study the gap produced a decrease in the inhibitory speed and in the latter an increase. This is not odd, as saccades have a different ecological relevance from hand and arm movements in primates. In fact, outside neurophysiology laboratories, they allow physical interactions with the environment, thus leading to material outcomes such as acquisition of food or tools.
Nevertheless, it is likely that the very general principles, not the fine details, of the genesis of eye and limb movements are rather similar.

\section{THE MOTIVATION TO ACT (EARLY “SHOULD-I-STAY-OR-SHOULD-I-GO" DECISION)}

The first step is represented by the motivation to act (early "should-I-stay-or-should-I-go" decision). This is determined by an evaluation process which is aimed at determining whether or not the individual's current needs are satisfied. The evaluative process can be primed because of a change in either the external environment (e.g., the sight of a cake) or the internal states (e.g., a sudden hungry feeling), or both (e.g., the sight of a cake prompts a hungry feeling). Thus, these first computations would evaluate whether or not the current state has to be changed to pursue a desire (e.g., eating) against several possible constraints (e.g., eating too much might cause weight gain). If the motivation is considered worthwhile then movement preparation will jump to the next stage, otherwise it will be canceled.

This is an essential process and one which is continuously performed by our motor system. In fact, in most places where we live, if not all, we are surrounded by tools whose sight automatically activates motor schemas that would normally be employed to interact with those objects. These actions are prompted by the features of the objects, the so-called affordances (Gibson, 
Table 1 | Summary of the main brain regions involved in each stage of the model describing the genesis of an arm goal oriented action, together with the most relevant references.

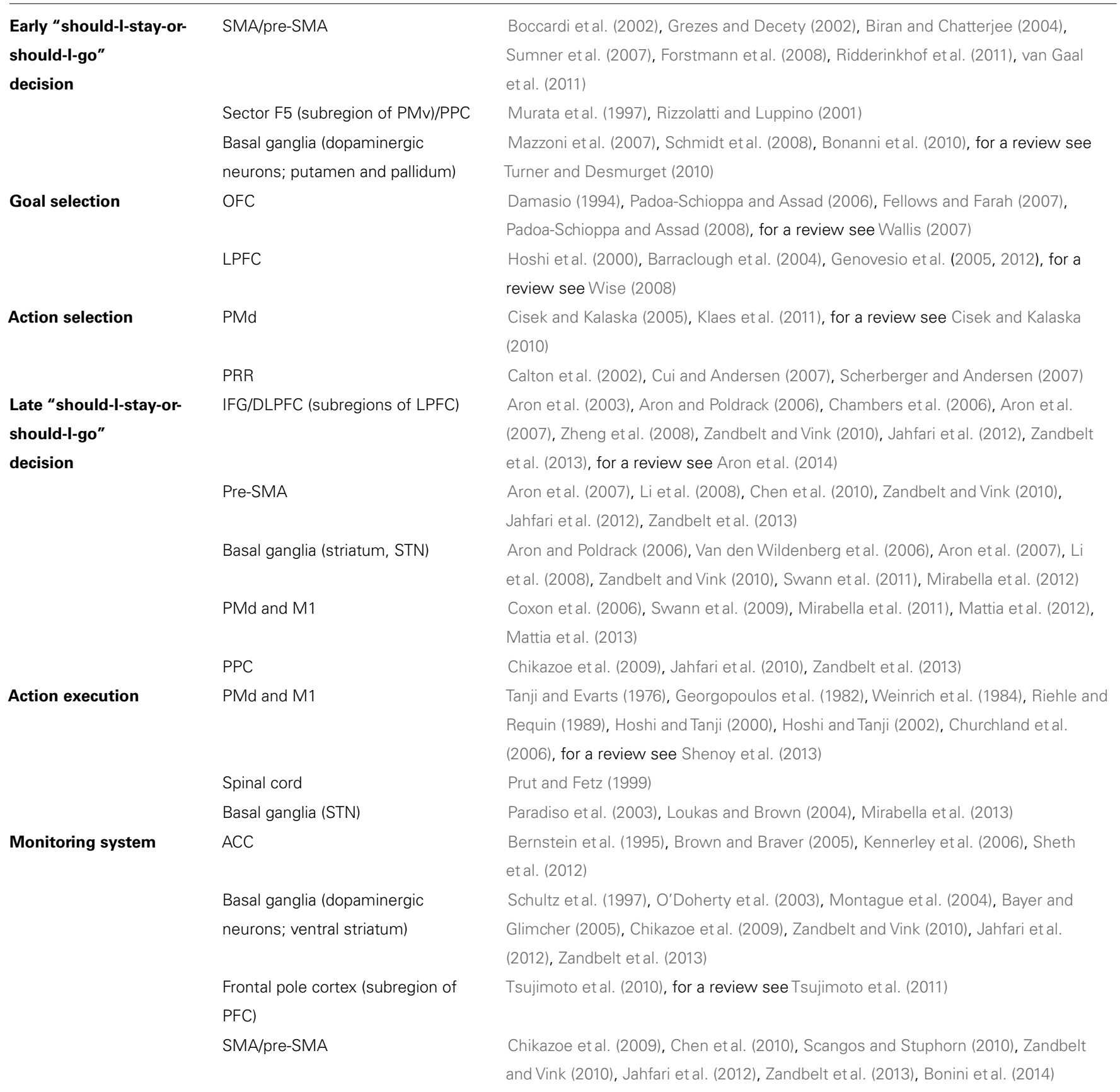

SMA, supplementary motor area; pre-SMA, pre-supplementary motor area; PMv, ventral premotor cortex; PPC, posterior parietal cortex; $L P F C$, lateral prefrontal cortex; DLPFC, dorsolateral prefrontal cortex; PMd, dorsal premotor cortex; PRR, parietal reach region; IFG, inferior frontal gyrus; STN, subthalamic nucleus; M1, primary motor cortex; ACC, anterior cingulate cortex; FPC, frontal pole cortex.

1979). It has been shown that even the simple observation of pictures depicting affordable objects (such as graspable objects) activates a sub-region of the medial frontal cortex, the supplementary motor area (SMA; see Figure 2), even when there is no requirement to actually act on those stimuli (Grezes and Decety, 2002). These stimulus-driven activations are rapid, involuntary, and unconscious.
Sumner et al. (2007), using subliminal stimuli to prime movements in a direction opposite to the requested one, showed that while control subjects were able to withhold unwanted actions, patients with microlesions of the SMA or the supplementary eye fields (SEFs) were impaired during the execution of hand or eye movements, respectively. They concluded that the SMA and SEF mediate automatic effector-specific suppression of motor plans. 


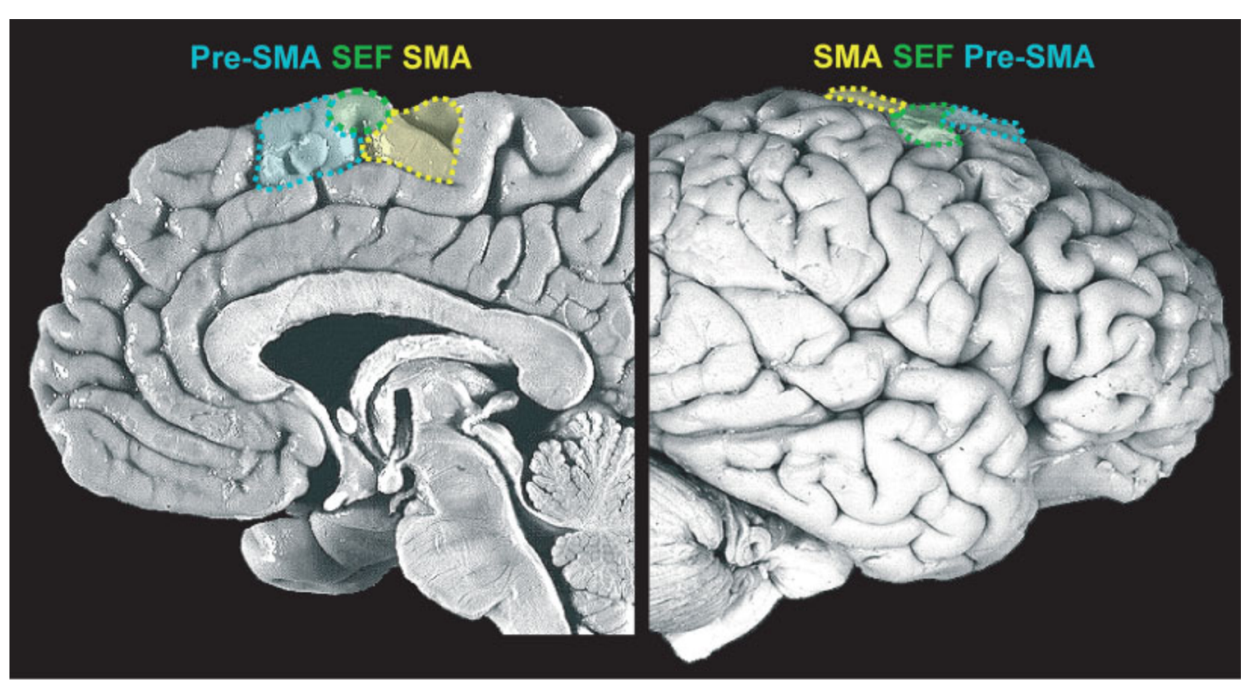

FIGURE 2 | Medial frontal cortex (details of the medial portion of Brodmann areas 6 and 8). Midsagittal view of the medial wall (left) and lateral prefrontal cortex (LPFC) surface (right), delineating the supplementary motor area (SMA), supplementary eye field (SEF) and pre-supplementary motor area (pre-SMA). Reproduced with permission from Ridderinkhof et al. (2011).
However, there is another way to look at those automatic activations: they might represent the activity of the network subserving the evaluative process which is at the base of the early "should-Istay-or-should-I-go" decision. In other words, affordances might increase the motivation to act, but to execute an action they have to be coupled with an internal state congruent with the primed action (e.g., the sight of a glass of water will prime the action if and only if an individual is thirsty; see Figure 1).

From this perspective the suppression of a triggered action might be seen not as an active process, but rather as an automatic consequence of the evaluative procedure. Using this framework, is possible to put forward a functional hypothesis underling two rare neuropsychological disorders, the alien limb syndrome and the utilization behavior. Both diseases are characterized by the fact that patients cannot resist objects' affordance, and they are automatically forced to perform stimulus-driven motor responses even when they do not need those objects (Humphreys and Riddoch, 2000). Patients with the alien hand syndrome perform involuntary actions with the limb contralateral to a focal brain lesion most frequently located in the medial frontal cortex, usually involving the SMA (Della Sala et al., 1991; Biran and Chatterjee, 2004). Patients suffering from utilization behavior compulsively grasp and use objects placed within their reach. This syndrome has been linked to bilateral damage to the medial frontal region involving the SMA, pre-SMA, and cingulate motor areas (Boccardi et al., 2002). Therefore, at least to some extent, the sites of the lesions causing those syndromes are largely overlapping, with the difference that in the former case it is located just in one hemisphere whereas in the latter case it affects both hemispheres. Possibly what is affected in both syndromes is the circuitry underlying the early "should-I-stay-or-should-Igo" evaluation, so that most stimulus-driven activations are no longer matched with our internal needs and thus they cannot be filtered out.
The discovery of the so-called canonical neurons in monkeys' lateral premotor area F5 may represent the neural mechanisms underlying responsiveness to object affordances (Murata et al., 1997). These neurons become active both when grasping an object and when seeing the same object without moving (Murata et al., 1997), and they are likely to feed the neural network of the medial frontal region which presides over the evaluation of whether to act. In fact, the lateral premotor area F5 receives projections from several regions of the posterior parietal cortex (PPC; Rizzolatti and Luppino, 2001), which is the end point of a crucial pathway for the visual guidance of actions toward objects, named the dorsal stream (Milner and Goodale, 1995). As such, PPC and F5 are likely to play a key role in visuomotor transformations, providing a neurophysiological correlate of stimulus-driven action affordance.

Several lines of evidence indicate that the fate of these activations might be decided in pre-SMA, which would act as a gate through which the available action affordances might be translated into actual actions (Ridderinkhof et al., 2011). First of all, a functional magnetic imaging (fMRI) study has shown that the strength of activation in pre-SMA covaries with the extent of inappropriate responses driven by stimulus-action association, i.e., the selection of appropriate action engages stronger activation of the pre-SMA in the face of many competing alternatives (Forstmann et al., 2008). Second, a strong negative correlation has been demonstrated between pre-SMA gray-matter volume and the inability to efficiently deal with competing response tendencies (van Gaal et al., 2011).

It would be very reductive to limit this first stage to the filtering of stimulus-driven action affordances and to the medial frontal regions. In fact, a role is surely also played by those brain regions that underlie arousal regulation (in particular the dopaminergic system), increasing or decreasing the readiness of animals to react. There are a wide variety of stimuli that can trigger arousal, from relatively simple sensory stimuli (e.g., the smell of blood or the 
sound of a mating call) to much more complex situations (e.g., the feeling of social exclusion or the feeling of being idolized). These signals prompt some basic instincts or learned memories which, according to the current contextual situation, might change the internal state of the animals, triggering an alert state. In these instances, animals do not yet have a goal, but they are more ready to act. The dopaminergic system is likely to play an important role in this stage of action genesis as witnessed by one of the symptoms of the most severe form Parkinson's disease (PD), the akinesia, i.e., the inability to initiate any goal-directed movement. Akinesia is not a pure motor disturbance, because it has been shown that in situations of great emergency (e.g., a missile attack, an earthquake) otherwise akinetic PD patients can show a sudden transient ability to move (paradoxical kinesia; Glickstein and Stein, 1991; Schlesinger et al., 2007; Bonanni et al., 2010). Evidently, when dopamine neurons are remarkably reduced in number, all behavioral options or internal mental states would appear to have the same value as the current state. In the face of this flat value function, the best choice for PD patients is to freeze. In extreme situations, salient stimuli may elicit stronger discharge of dopaminergic cells than normal, driving the patient to act. Recently, Mazzoni et al. (2007) suggested that even in less dramatic situations the slowness of movements (bradikinesia) in PD patients may be attributable to an improper evaluation of movement energy costs. In these experiments, PD patients and healthy controls were asked to move their arm to a previously specified target at different speeds a given number of times. Both were able to make required movements with the same accuracy, but PD patients needed significantly more trials before reaching the required number of repetitions. As the accuracy of patients was the same as that of controls, authors concluded that the loss of dopamine did not cause bradykinesia through a speed-accuracy trade-off. Rather, it affected decision-making through a faulty evaluation of the costs of movements, i.e., allocation of the correct amount of energy to meet the demands of the task. In other words, PD weakened the key link between motivation and movement gain. In fact, the dopaminergic system has been shown to play a major role in reward-dependent learning (e.g., Schultz et al., 1997; Montague et al., 2004).

Other evidence indicates that the regulation of action motivation based on previous experiences is one of the main functions of the basal ganglia, not just of the dopaminergic system (Turner and Desmurget, 2010). This hypothesis is supported by one symptom which often accompanies focal damage of the basal ganglia, the so-called auto-activation deficit or abulia (Habib, 2004), in which patients suffer from a marked deficit in motivation to perform spontaneous acts despite an absence of overt motor impairment. In particular, Schmidt et al. (2008) asked patients suffering from auto-activation deficit, due to bilateral lesions of the putamen or pallidum, to control grip forces in response either to explicit sensory instructions or to monetary incentives. Although they fully understood the instructions, patients were capable of modulating their movement only in the former, not in the latter, condition.

All in all, it is clear that there are several brain regions that regulate the willingness to act. This early "should-I-stayor-should-I-go" decision should play a key role because it would activate (or stop) the chain of other decisions that will potentially lead to action execution. While many other subsequent stages might be performed in parallel, this first one is likely to be a standalone process during which no actions are planned and thus there is no need for any neural signal to inhibit them.

\section{GOAL SELECTION}

Once performance of an action is considered worthwhile, because a need has to be satisfied, the next set of decisions will be devoted to the selection of the most opportune goal among the several different alternatives usually available (e.g., to satisfy the feeling of hunger we might decide to eat the cake in front of us or to leave the room and go to a restaurant). This process possibly entails two stages: first, values are assigned to each available option and subsequently a decision is taken weighting these values according to the behavioral context (e.g., Glimcher et al., 2005). Even though this schema is conceptually logical, it is very unlikely that neural processing would be strictly organized in distinct serial stages. As depicted in Figure 1, it is very likely that multiple potential goals are simultaneously represented. To each goal a given value would be assigned and weighted according to the situation. As multiple goals cannot be pursued at the same time, these representations probably start to compete with each other, perhaps through mutual inhibition (Figure 1). This competition can be biased by several factors which ultimately influence the expected outcome of the action ${ }^{1}$. In this review I will argue that these biasing signals might come from the brain regions of the "monitoring system," i.e., the system that evaluates and stores the outcome of past actions (see Figure 1 and see The Monitoring System). Value assignment and the competition might occur at the same time, probably in different sectors of the prefrontal cortex (PFC). PFC is not a homogeneous structure but is composed of multiple areas that differ in terms of cytoarchitecture and anatomical connections with other areas (Petrides and Pandya, 1999, 2002; Figure 3). Thus, it is likely that each area might perform its own function; however, their exact roles are not yet clear. Part of the reason is that the PFC contributes to a bewildering array of functions (Wise, 2008).

Nevertheless, converging evidence argues that orbitofrontal cortex (OFC) plays a key role in linking stimuli to their values (for a review see Wallis, 2007). Indeed, lesions to the OFC impair choice behavior, leading to unreliable choices (e.g., Damasio, 1994; Fellows and Farah, 2007) or abnormal gambling (e.g., Camille et al., 2004; Koenigs and Tranel, 2007). Padoa-Schioppa and Assad (2006), recording single-units in monkeys, demonstrated that a particular class of neurons of the OFC ("chosen neurons") encode the subjective value of two different drinks (juice and water) irrespective of their taste, volume or the action that needs to be taken to obtain them. Thus these cells encoded value per se, allowing a comparison for qualitatively different goods. Later on, Padoa-Schioppa and Assad (2008) also showed that the discharge of these neurons was independent of the presence of other goods. These findings indicate that the OFC produces stable value representations, i.e., a key trait of choices, because it allows abstract comparisons such as

\footnotetext{
${ }^{1}$ An exception to this model might occur when the number of potential goals exceeds the capacity of working memory. In these instances goals might be processed differently.
} 

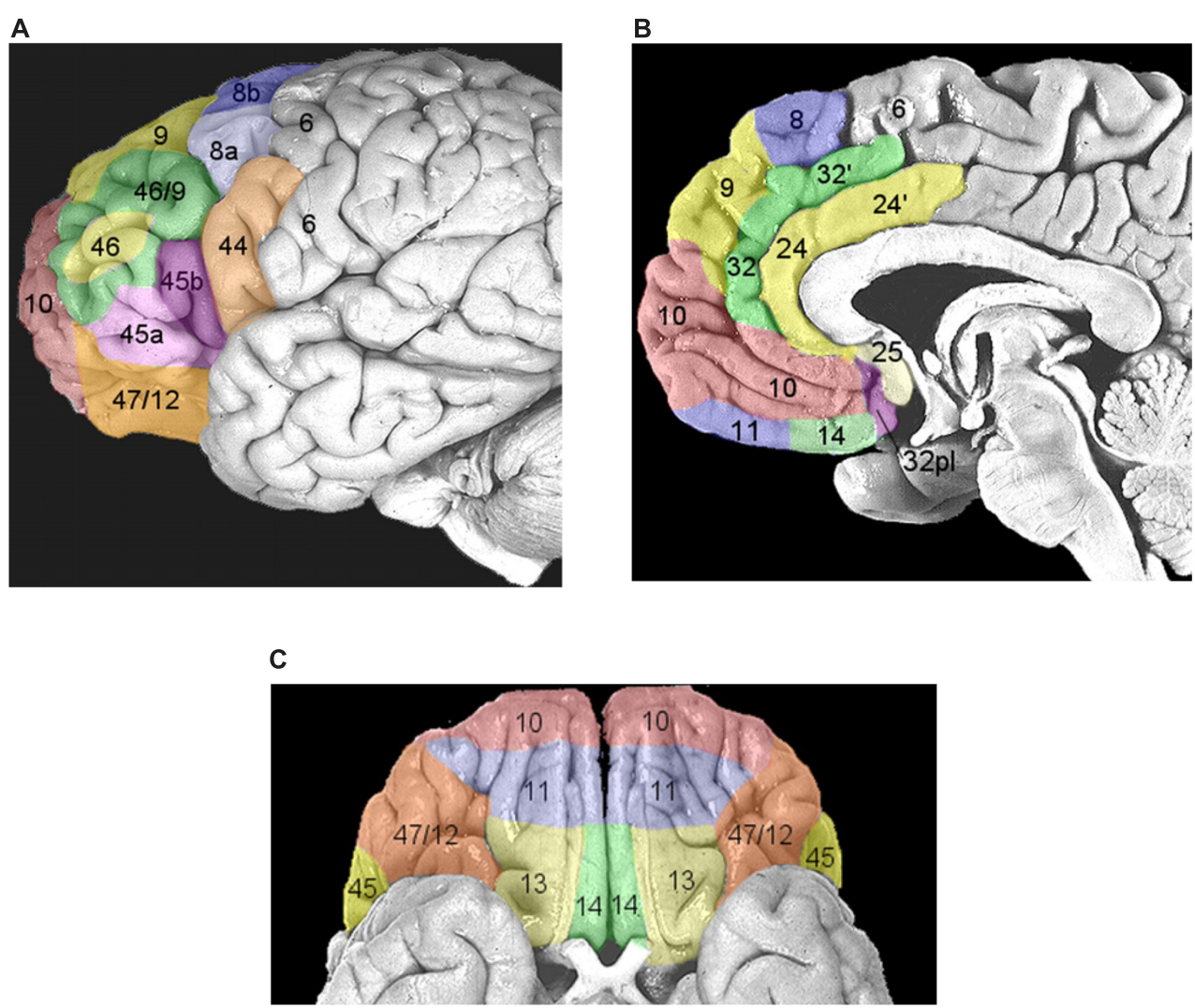

FIGURE 3 | Areas composing the prefrontal cortex (PFC) according to the parcellation of Petrides and Pandya $(1999,2002)$. (A) Areas of the lateral PFC. The inferior frontal gyrus (IFG) corresponds to Brodmann area (BA) 44 , or pars opercolaris, to BA45, or pars triangularis, and to BA47/12, or pars orbitalis. The dorsolateral prefrontal cortex (DLPFC) corresponds to BA9/46 and BA46. (B) Areas of the medial wall of the PFC. The anterior cingulate cortex (ACC) corresponds to BA32, BA24, and BA25 (dorsocaudal portions are indicated with a hyphen). BA10 corresponds to the frontopolar cortex. The orbitofrontal cortex (OFC) corresponds to BA11 and BA14. (C) Areas of ventral orbital surface of the PFC. BA10 corresponds to the frontopolar cortex. Area BA47/12 corresponds to the pars orbitalis of the IFG. The OFC corresponds to BA11, BA13, and BA14. Reproduced with permission from Ridderinkhof etal. (2004). transitivity between different goods which are not available at the same time.

On the other hand, to select properly the more appropriate goal, the values of the options must be evaluated in light of the situation which an animal has to face (e.g., if an animal is starving even a non-preferred food represents a good choice). Therefore, to represent value efficiently in different situations, a neuronal representation should flexibly adapt to the current context. These computations are probably carried on in lateral prefrontal cortex (LPFC). Several studies have shown that neural activities of those areas specify the so-called task sets. A task set is a configuration of perceptual, attentional, mnemonic, and motor processes that is actively maintained to perform a given task. It specifies the rules needed to solve the specific task, but it is independent of the stimuli as long as they have to be processed in the same way. Therefore it is not surprising that the PFC contributes to an enormous array of functions ranging from selective attention (Lebedev et al., 2004) to working memory (Funahashi et al., 1989), problem-solving strategies (Genovesio et al., 2005) and categorization of sensory stimuli (Freedman et al., 2002). This list is by no means exhaustive, but it is intended to give an idea of the several kinds of knowledge that are processed in the LPFC. Recently, a series of studies has provided evidence about the way in which this array of cognitive processes can be combined to produce sophisticated behavior (Genovesio et al., 2005, 2006, 2012). The logic underlying all these experiments was that of setting tasks which require several kinds of long-term and shortterm knowledge, while recording single-unit activity in the LPFC. For instance, Genovesio et al. (2005) trained monkeys to make a saccade to the left, right, or upward direction in response to a visual object, depending on the cue and on the goal that had occurred on the previous trial (Figure 4). This way the monkeys could not learn a fixed stimulus-response association. Instead, they had to adopt repeat-stay and change-shift strategies, i.e., if the cue was the same as in the previous successful trial the monkeys repeated the response, while if the cue was different they had to change their response. Genovesio et al. (2005) found that the activity of some single-neurons in the dorsolateral prefrontal cortex (DLPFC) represent different strategies, that is, special kinds of abstract rules acquired on the basis of task performance history. Similarly, Barraclough et al. (2004) found that DLPFC neurons encoded monkeys' past decisions and payoffs, providing crucial 
A

Previous trial

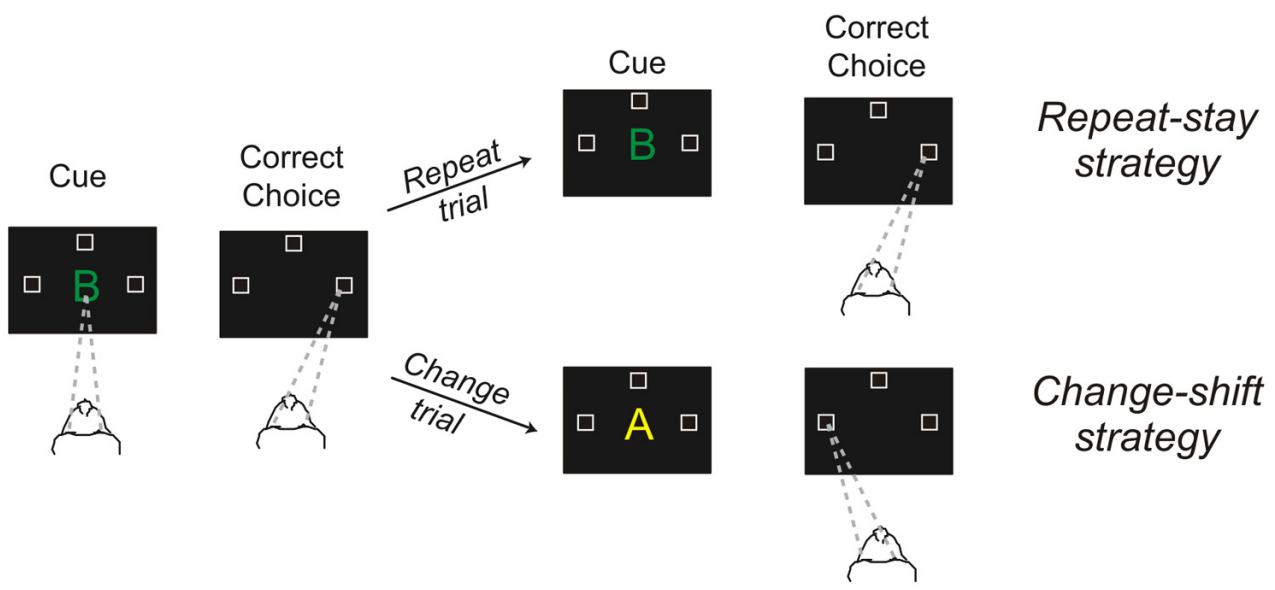

B

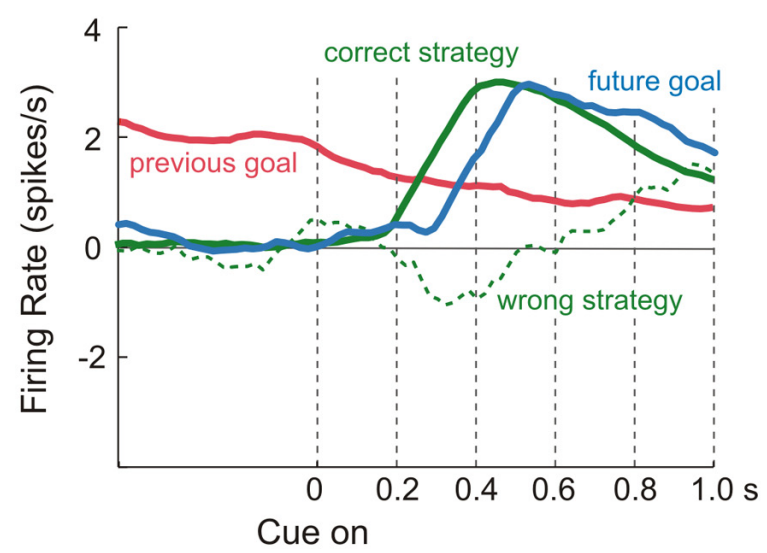

FIGURE 4 | Role of dorsolateral prefrontal cortex during a "strategy task." (A) Temporal sequence of the visual displays during the task and behavioral responses required of the monkeys. Each trial began when monkeys fixated on a spot at the center of the display (gaze direction is indicated by the dashed lines). After a delay, a cue appeared. When it disappeared, the monkeys had to make a saccadic eye movement to one of three positions (unfilled squares). Monkeys were required to remember both the cue and the goal of the trial just performed because the response on the next trial (current trial) depended on the previous choice, i.e., if the cue was the same as in the previous successful trial, the monkeys repeated the response (repeat trial), while if the cue was different they had to change their response (change trial). Thus, monkeys were forced to change strategy according to the past trial history, adopting either a repeat-stay strategy or a change-shift strategy (B) Neural activity reflecting the previous goal (red), the future goal (blue), the correct strategy (solid green line), and the wrong strategy (dashed green line). Previous-goal signal decreased after cue onset as the signals for the correct strategy and future goal increased. In contrast, when monkeys chose the wrong strategy, a weak or absent strategy signal. occurred during the time of goal selection. Reproduced with permission from Wise (2008). signals to update estimates of expected reward. Thus, according to the authors the activity of these cells subserves the optimization of decision-making strategies. Other neurons in the same area represented fixed stimulus-response mappings learned previously (Hoshi et al., 2000).

All in all it seems that the different subregions of the LPFC process several types of knowledge and, taking into account the context in which the animals operate and the outcome of the action performed, allow the performance of non-routine, i.e., flexible, behaviors (Wise, 2008). Clearly such complex elaborations cannot be done in isolation. In fact, it has been recently proposed that information about several different metrics of available resources in the surrounding environment (e.g., numerosity, duration, distance) are provided to the LPFC by the PPC (Genovesio et al., 2014). Certainly, along the fronto-parietal network attentive signals flow bidirectionally, allowing the selection of salient stimuli during visually guided movements (e.g., Lebedev and Wise, 2001; for reviews see Corbetta and Shulman, 2002; Reynolds and Chelazzi, 2004). Two other relevant sources of information are the OFC, which, as described at the beginning of this paragraph, delivers knowledge about the value of the stimuli, and the anterior cingulate cortex (ACC) and/or the pre-SMA, which 
deliver signals dealing with prediction of expected outcome (see The Monitoring System). In addition, sensory areas could also contribute to this process. For instance, it has been found that activity of neurons in macaque area V4 can underlie the selection of elemental object features and their translation into a responserelated format that can directly contribute to the control of the animal's actions (Mirabella et al., 2007).

These are just a few examples; many cortical and subcortical structures are connected with the PFC, which is optimally situated to gather and synthesize information to select the more appropriate goal, and the best task set to achieve it in a given context.

\section{ACTION SELECTION}

Even though goal and strategies are selected in the PFC, there are several lines of evidence indicating that choosing between alternative actions to achieve an identified goal and the generation of specific motor commands (i.e., motor plans) are accomplished by neural populations in the dorsal premotor cortex (PMd; Cisek and Kalaska, 2005; Klaes et al., 2011), in the primary motor cortex (Tanji and Evarts, 1976) and in the so-called parietal reach region (PRR), a subregion of the PPC (Cui and Andersen, 2007; Scherberger and Andersen, 2007). Therefore goal selection and the selection of movements to reach them are two separate processes that, to some extent, are likely to occur one after the other.

As most goals can be achieved in any of several ways, multiple potential actions are possibly represented at the same time and start to compete for implementation ${ }^{2}$. That this was the case was demonstrated by Cisek and Kalaska (2005) in a seminal experiment. While recording from PMd, they set a task in which two spatial cues indicated two opposite potential reaching actions. After a delay, a non-spatial cue specified the correct choice (Figure 5). Soon after the presentation of the two cues, the neural activity of PMd specified both directions of potential reach targets simultaneously. When information for selecting the correct action became available, its neural representation was strengthened while the other was suppressed.

On these grounds, the authors hypothesized that PMd prepares multiple actions in parallel and selects between them through a process of biased competition taking place within the same neural substrate that guides the execution of those actions (Cisek, 2006; Cisek and Kalaska, 2010). Thus, the mechanism of action selection is likely to occur in a similar way to that underlying goal selection (Figure 1).

Dorsal premotor cortex is not the only site where multiple potential actions can be represented. In fact, it has been shown that if a monkey is presented with a spatial target, but not instructed about whether an arm or a saccadic eye movement is required, neurons begin to discharge simultaneously in different regions of the PPC, the lateral and the medial intraparietal sulcus (LIP and MIP, respectively; Calton et al., 2002; Cui and Andersen, 2007). These discharges represent the simultaneous coding of saccade

\footnotetext{
${ }^{2}$ As previously stated for the representation of multiple goals, even in this instance the representation of multiple potential movements may be limited by the capacity of working memory.
}

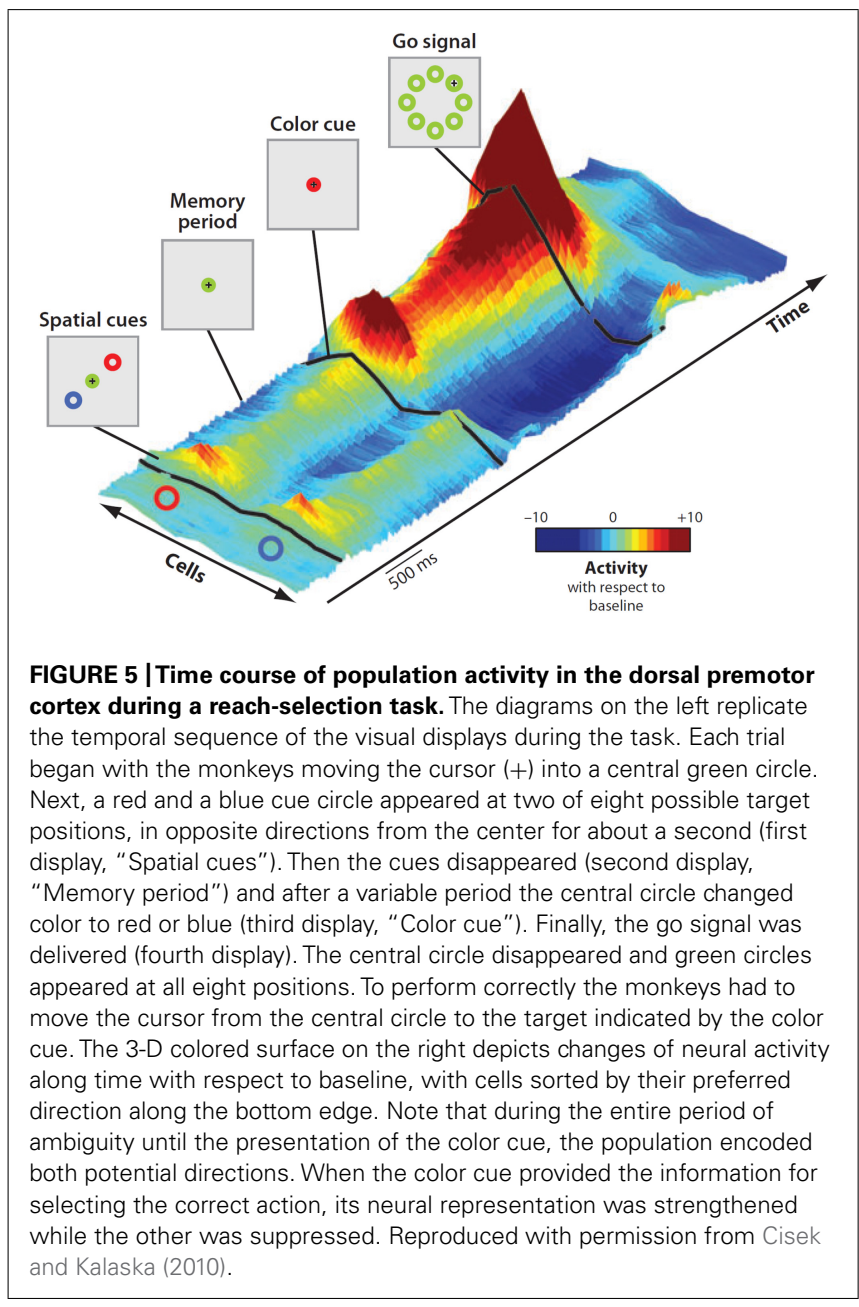

and reach plans. Later, if an arm movement is cued (Calton et al., 2002) or freely chosen (Cui and Andersen, 2007), the activity of MIP becomes stronger than that of LIP, and vice versa if a saccade is instructed or chosen.

Recently, Klaes et al. (2011) confirm and extended these findings by demonstrating that in situations of uncertain choice, the frontoparietal reach areas (PRR and PMd) construct all potential motor alternatives. However, Klaes et al. (2011) made a further step showing that that potential actions were also biased by the monkeys' subjective desirability, confirming the model proposed by Cisek (2006).

All in all, these findings seem to indicate that in the frontoparietal reach areas there is a continuous and simultaneous processing of multiple movement options. Possibly, as some computational models predict (e.g., Smith and Ratcliff, 2004), neural activity related to response choices, i.e., the motor plans, builds up in separate accumulators as a function of the evidence for or against them until one reaches a threshold, winning the competition.

What a motor plan is or what it represents is a very debated issue. It is generally accepted that the motor plan is formed in the premotor cortex and in M1 (Tanji and Evarts, 1976; Weinrich et al., 1984), but what is encoded by the neural activity of the motor cortices and how it relates to movement activity are matters of some 
controversy. It has been proposed that the discharge of single-unit might represent a subthreshold version of movement (Tanji and Evarts, 1976) or that population activity represents some movement parameters (e.g., reach direction and distance Georgopoulos et al., 1982; Riehle and Requin, 1989) or even the combination between information about the target and about the effector used (Hoshi and Tanji, 2000, 2002). However, all these approaches have been proven to be inconsistent or equivocal in fully explaining the activity in the motor system (for a review see Shenoy et al., 2013).

An alternative proposal is that this activity reflects a mix of signals: "some will be outputs to drive the spinal cord and muscles, but many will be internal processes that help to compose the outputs, but are themselves only poorly described in terms of the movement" (Shenoy et al., 2013, p. 339). In other words, the activity that precedes a movement would represent the initial state of a dynamic system which will determine the temporal patterns needed to drive actions. Under this hypothesis the motor plan does not explicitly represent movement parameters; it is still closely related to movement activity, but the reciprocal relationship is not transparent at the level of the individual cells. This framework seems to reconcile several past, apparently contradictory, findings and to provide a wider comprehension of the functioning of the cortical motor system (Shenoy et al., 2013). One possible limit of the dynamic system hypothesis, that future studies will have to overcome, is that most of the data come from experiments in which there is a delay between the appearance of the target and the go signal (delayed-reach task, e.g., Churchland et al., 2006). Clearly, to be fully validated the hypothesis should be tested in tasks featuring performance or inhibition of reaching movements in different contexts (but see Mattia et al., 2013).

In conclusion, the same substrates where the action selections occur are also those that are used to prepare and guide the execution of the movement that is ultimately selected.

\section{THE FINAL EVALUATION (LATE “SHOULD-I-STAY-OR-SHOULD-I-GO" DECISION)}

By the end of the previous stages, what had been evaluated as the best action to achieve the desired goal is planned, but before the corresponding motor plan can be executed it has to pass a further final check (late "should-I-stay-or-should-I-go" decision). This is a fundamental step, because from the moment at which the decision to act has been taken to the time when the motor output is about to be generated, the continuous flow of information might signal that something has changed in the external environment, in the internal states or in both. These changes might impact on the previous evaluations as the selected action might turn out to be no longer appropriate (Haggard, 2008). A common experience might be that of a person about to cross a road but, just before stepping onto the road, he hears the sound of an ambulance siren clearly approaching. In the most common instance, the person would halt his step to avoid being hit. However, the evaluation might be radically different if his/her child is already in the middle of the road. In the former case suppressing the action is clearly the most valuable decision but, in the latter, the risk of losing the parental investment might trigger the person to act even faster in order to secure the child. The evaluation could be also influenced by endogenous signals, for instance the same person in the above example could take the risk of crossing the road because he suddenly remembers that his plane is leaving.

Computationally this last check could be realized by comparing the output of a predictive forward model with a goal description (e.g., see Wolpert and Miall, 1996). When the mismatch between the predicted result and the goal becomes too large, i.e., the action is unlikely to allow the achievement of the desired result, the pending action is canceled. There are several tasks that are currently exploited to study the inhibitory function and each has some advantages over the others (for a review see Ridderinkhof et al., 2011); however, in order to design a potential neural network capable of augmenting inhibition of pending actions, I will focus on the stop-signal paradigm (Logan and Cowan, 1984). There are two reasons to choose this as a paradigmatic task. Firstly it is the only one which allows study of the suppression of ongoing movements, and secondly it has been widely used exploiting several effectors (the eyes, e.g., see Hanes and Schall, 1996; the finger, e.g., see Logan and Cowan, 1984; the arm, e.g., see Mirabella et al., 2006); thus a wealth of data are available. The stop-signal (or countermanding) paradigm probes a subject's ability to withhold a planned movement triggered by a go signal when an infrequent stop-signal is presented after a variable delay (see Figure 6A).

Starting from the behavioral performance during the countermanding task it is possible to yield an estimate of the duration of the suppression process [stop-signal reaction time (SSRT); Logan and Cowan, 1984; Band et al., 2003; Boucher et al., 2007]. The SSRT is a key behavioral parameter for uncovering the neural substrates of inhibition. In fact, those brain regions showing a change in activity when a movement is produced with respect to when it is suppressed, and where the onset of this shift precedes the end of the SSRT, can be assumed to be causally related to the suppression process.

Thus the stop-signal task allows to study of the way subjects react to an unexpected imperative stop instruction. This is referred to as "reactive inhibition." At the same time, this approach also allows assessment of changes in the response strategies of individuals embedded in such an experimental context. In fact, the rules of the countermanding task create a conflict on all no-stop trials because subjects are instructed to move as fast as possible, but, at the same time, they tend to delay movement initiation to wait for the occurrence of a possible stop-signal. As a consequence, healthy subjects had longer RTs when executing go-trials intermixed with stop-trials than when executing go-trials alone (e.g., Mirabella et al., 2006; Verbruggen and Logan, 2009). In addition, the occurrence of stop trials induces a lengthening of the RTs of responses produced in the immediately subsequent no-stop trials (Mirabella et al., 2006, 2012; Verbruggen and Logan, 2009; Zandbelt and Vink, 2010). This form of control over response execution in anticipation of known task demands, driven by endogenous signals, i.e., the awareness of the possible presentation of stop-signals, has been called "proactive control/inhibition." In the following, I will describe results mainly related to reactive inhibition, while I will mainly focus on proactive inhibition in the next paragraph where 


\section{A}
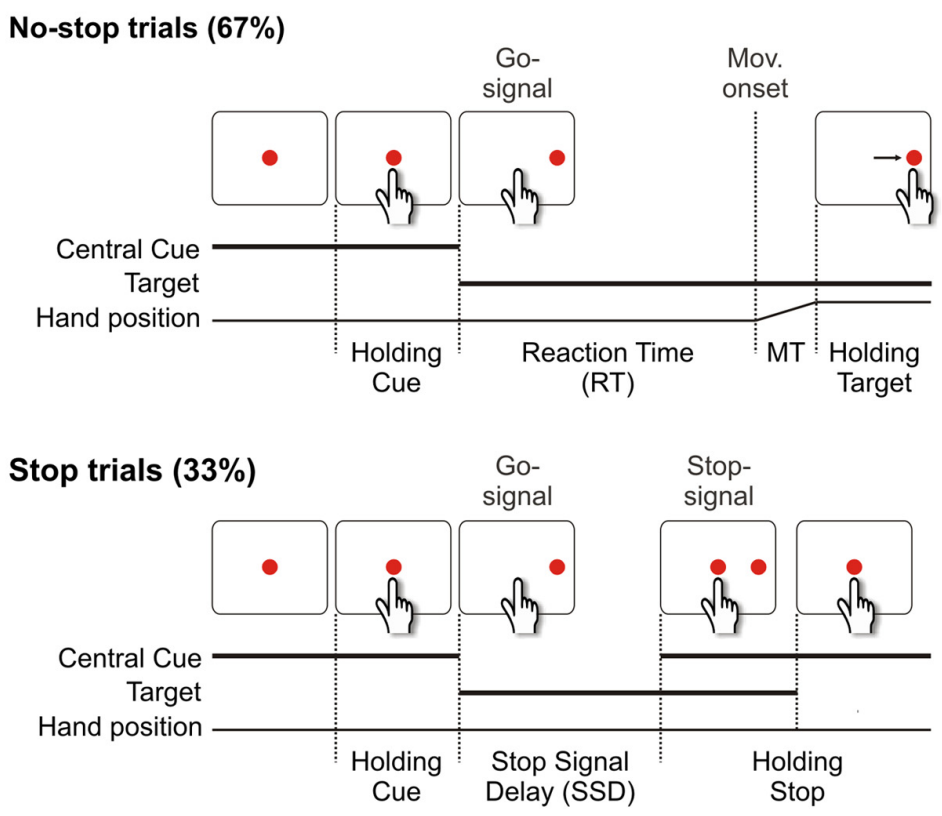

B
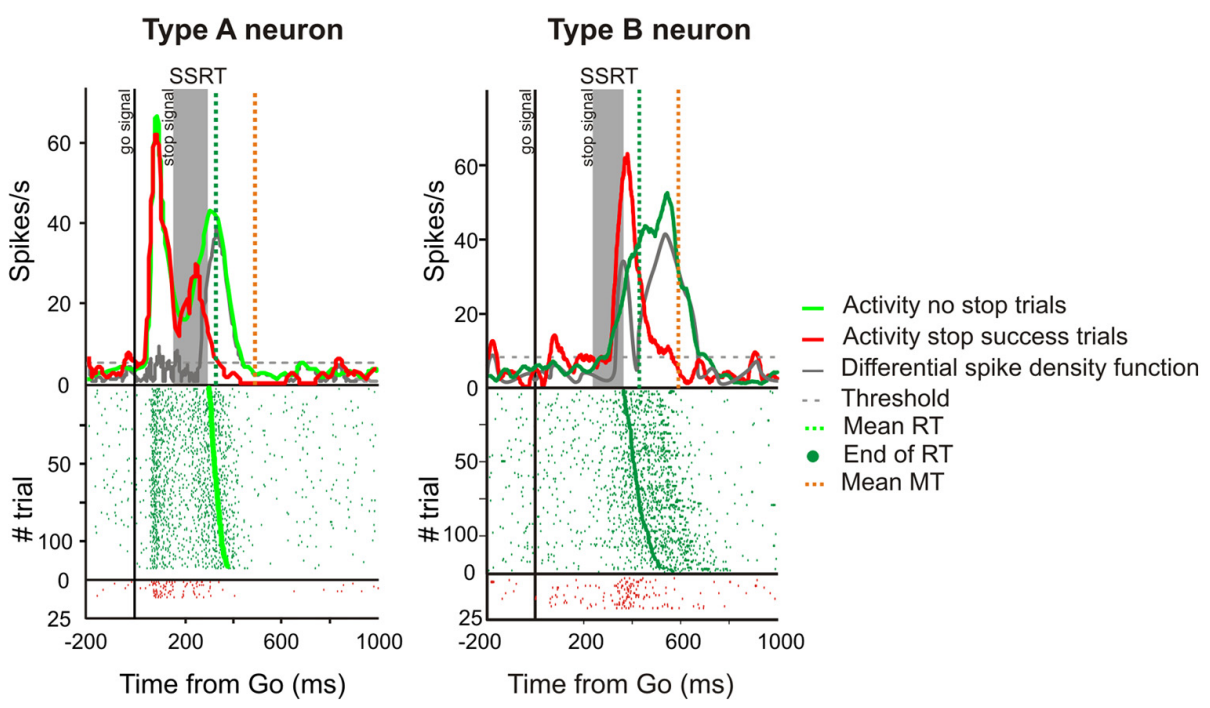

FIGURE 6 | Causal role of neurons of the dorsal premotor cortex (PMd) in reactive inhibition. (A) Temporal sequence of the visual displays for no-stop and stop trials in the reaching version of the countermanding task. All trials began with the presentation of a central stimulus. After a variable holding delay (500-800 ms) it disappeared and simultaneously a target appeared to the right, acting as a go-signal. In the no-stop trials subjects had to start a speeded reaching movement toward the peripheral target. Randomly, on a fraction of interleaved trials (33\%), the central stimulus reappeared after variable delays (SSDs), instructing subjects to inhibit movement initiation. In these stop trials, if subjects countermanded the planned movement keeping the arm on the central stimulus the trial was scored as a stop-success trial. Otherwise, if subjects executed the reaching movement the trial was scored as a stop-failure trial (not shown).

Reproduced with permission from Mattia et al. (2012). (B) Changes of activity driven by the stop-signal onset in PMd neurons modulated during the preparation of the movement. In each panel the upper graph represents the average spike density function while the lower graph shows the raster plots of neural activity in no-stop trials (green tick-marks) and stop-success trials (red tick-marks). Neural activity was always aligned to the go-signal onset (first vertical line). The gray band represents the estimated duration of the stop-signal reaction time (SSRT) in that session. The gray line represents the differential spike density function, while the dashed gray line represents the threshold value for significant divergence. The green and the orange vertical dotted lines in the top panels indicate the average RT and the average end of MT, respectively. The green dots in the rasters represent the end of the RTs. On the right, the activity of a representative "type $A$ " countermanding neuron is shown. In this cell, neural activity during stop-success trials (red line) initially resembles that of no-stop trials (green line) but, with a delay after the stop-signal presentation, it suddenly starts to decrease and the differential spike density function crosses the threshold $34.4 \mathrm{~ms}$ before the end of the SSRT. On the left, the activity of a representative "type B" countermanding neuron is shown. In this instance, the activity in stop-success trials increases after stop-signal presentation with respect to that recorded during no-stop trials $39.9 \mathrm{~ms}$ before the end of the SSRT. Therefore both these two types of neurons exhibit a modulation of activity sufficient to control the suppression of an ongoing arm movement. 
I will deal with the monitoring system (but see also Action Execution). Clearly, all proactive strategies necessarily derive from these computations. This does not mean the neural substrates of reactive and proactive inhibition have to be different. In fact, it has been shown that there is an overlap between them (e.g., Jahfari et al., 2012; Zandbelt et al., 2013; for a review see Aron, 2011); however, reactive and proactive inhibition might derive from two conceptually different modules (the late "should-I-stayor-should-I-go" decision module and the monitoring system; see Figure 1).

Variants of the stop-signal task have been used several times in association with different techniques [e.g., single-unit recordings, fMRI, electroencephalographic scalp recordings (EEG), intracranial electroencephalographic recordings (iEEF), lesions, transcranial magnetic stimulation (TMS), deep brain stimulation (DBS)], different effectors (the eyes, the arm, and the fingers), and different pathologies (e.g., PD, attention deficit and hyperactivity disorder, Tourette syndrome, obsessive-compulsive disorder). From this large number of studies, a network of brain regions that seem to be involved in implementing inhibition has been identified. To this network belong both cortical and subcortical structures, that largely overlap with those involved in movement generation, planning, and even movement execution.

One of the prefrontal regions that more frequently has been reported to have an inhibitory role is the inferior frontal gyrus (IFG), especially in the right hemisphere (see Aron et al., 2014 for a recent review). For instance, Aron et al. (2003) have shown that humans with a lesion to the right, but not to the left IFG exhibit longer SSRTs than healthy subjects. Furthermore they showed that the lengthening of the SSRT was proportional to the extent of damage in the right IFG. Less frequently, the DLPFC has also been claimed to participate in this executive function (e.g., Zheng et al., 2008). However, its role is controversial. In fact, while Zheng et al. (2008) showed that individuals who were more proficient at inhibition had a greater activation in DLPF, Chambers et al. (2006) found that temporary deactivation of the same region, with repetitive TMS, did not significantly alter the speed of inhibition.

Imagining studies revealed the involvement in this form of inhibition of the pre-SMA (e.g., Aron et al., 2007; Li et al., 2008; Zandbelt and Vink, 2010; Jahfari et al., 2012) as well as of the striatum (Li et al., 2008; Zandbelt and Vink, 2010). Even in this instance the exact role of these structures is unclear and debated. Li et al. (2008) compared the fMRI brain activation of individuals with short versus long SSRTs who were identical in all other aspects of stop-signal performance. Their aim was to isolate the neural correlates of response inhibition from those of response monitoring and/or attentional control. Under these experimental conditions, Li et al. (2008) found that the caudate head had greater activation in individuals with short than with long SSRT, and the extent of its activation was positively correlated with activity in the pre-SMA. In contrast, Scangos and Stuphorn (2010), by recording singleneuron activity of SMA and pre-SMA of monkeys during an arm countermanding task, found that these cells could not contribute directly to response inhibition as most of them modulate after the SSRT. Instead, the majority of neurons signaled expectation of reward, as they were modulated by the amount of expected reward.

Another basal ganglion implicated in stopping ongoing actions is the subthalamic nucleus (STN; see Aron and Poldrack, 2006). Mirabella et al. (2012) demonstrated that bilateral STN DBS selectively improves inhibitory functions as its electrical stimulation significantly shortened the SSRT, but did not influence the RTs of no-stop trials. These results agree with those of Van den Wildenberg etal. (2006) and Swann et al. (2011), but not with those of Li et al. (2008). In the above-described study, they found that neither the STN nor the IFG were active during reactive stop and thus concluded that both structures had a role in attentional monitoring of the stop-signal. Similarly, Zandbelt and Vink (2010) did not find evidence of STN activation during movement cancelation.

Finally, two other areas have been found to be involved in inhibitory function, i.e., the PMd (Mirabella et al., 2011; Mattia etal., 2012, 2013) and the primary motor cortex (M1; Coxon etal., 2006; Swann etal., 2009; Mattia etal., 2012). Mirabella etal. (2011) showed that about 30\% of PMd cells changed their discharge before the end of the SSRT when the monkey had to stop a reaching movement. Thus these neurons seem to be causally involved in reactive inhibition (see Figure 6B). These findings were confirmed and extended by a subsequent study (Mattia et al., 2012), in which epicortical event-related potentials (ERPs) were recorded from the lateral surface of the fronto-temporal lobes of epileptic patients performing the countermanding task. It was found that an ERP complex was selectively expressed before the end of the SSRT in M1, and in the premotor cortex (Figure 7). Thus, Mattia et al. (2012) deduced that motor cortices are causally involved in inhibitory control. In conclusion, even though the exact role of each of the brain regions involved in the stop task remains controversial, it clearly emerges a considerable overlap between brain region subserving the preparation of an action and its suppression (see Table 1).

To summarize, the late "should-I-stay-or-should-I-go" decision represents a hinge of our goal-directed behavior because it allows crucial, last-minute changes of strategies when the cost of an action overcomes the benefits. Unlike the early "shouldI-stay-or-should-I-go" decision, which does not require active suppression, in this instance some neural signals aiming to halt the activity linked to the ongoing action have to be produced by the nervous system (Figure 1). Given this, it should not come as a surprise that such a large network of brain regions has to be involved.

\section{ACTION EXECUTION}

Once the last check is passed, the motor commands are sent to the spinal cord, activate the muscles, and produce the movement. It must be remarked that it is very unlikely that even the spinal cord would be a passive relay of "higher directives." In fact, Prut and Fetz (1999) have shown that spinal interneurons show pre-movement activity during a delayed task, similarly to PMd (Wise et al., 1983). This indicates that, at least under some experimental conditions, movement preparation may occur simultaneously over widely distributed regions, including spinal levels. 


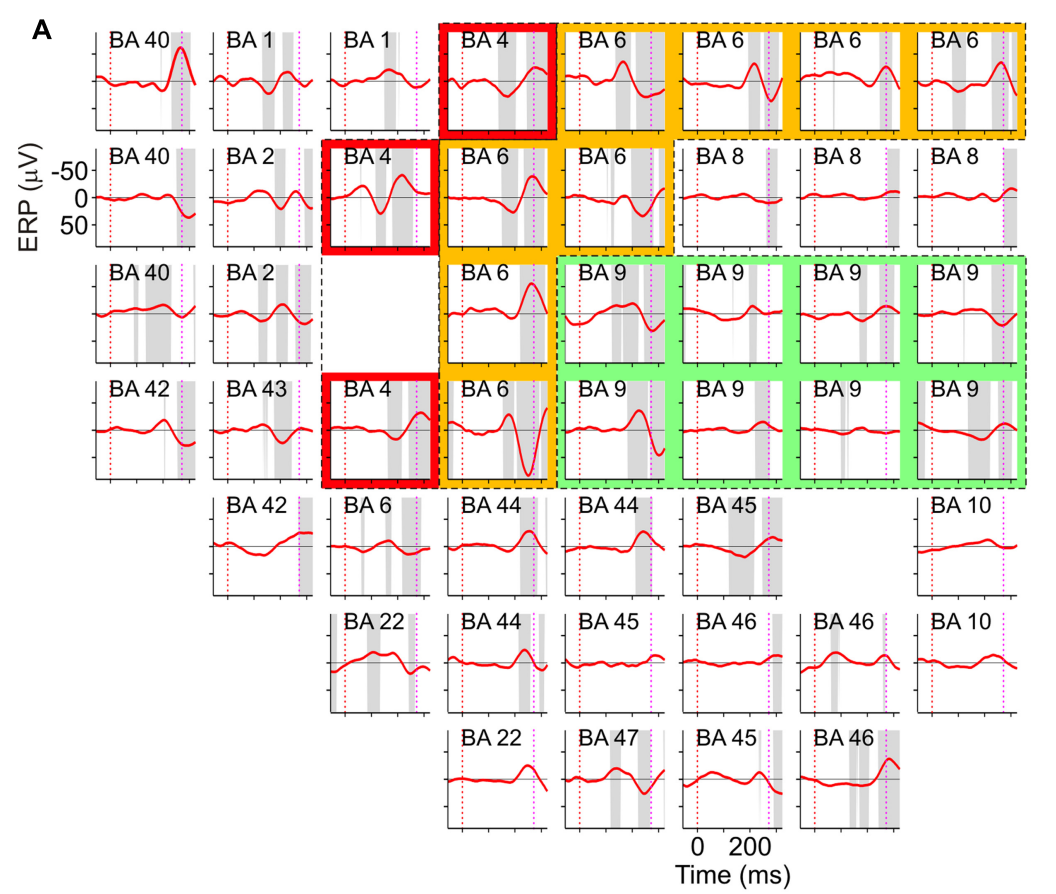

B

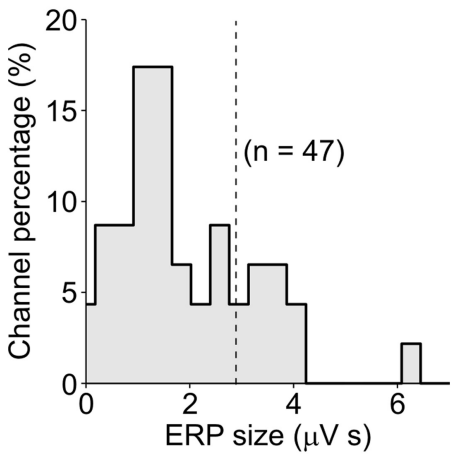

C

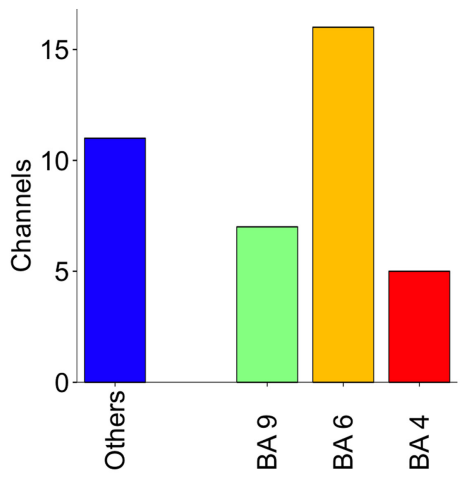

FIGURE 7 | Spatiotemporal distribution of stop-event related potentials (ERPs) in successful-stop (SS) trials. (A) Average stop-ERPs (solid red curves) of SS trials centered on stop-signal appearance corresponding to the selected channels for one pharmacoresistant epileptic patient. Gray areas, time intervals at which the stop-ERP was significantly different from 0 (Wilcoxon signed-rank test, $P<0.01$ ). Subplot labels: Brodmann areas (BAs) over which electrodes were positioned. Colored areas: electrodes placed over the primary motor cortex (red, BA4), the premotor cortex (yellow, BA6) and the DLPFC (green, BA9). (B) Histogram of the stop-ERP sizes of panel (A). Stop-ERP sizes were computed as the integral of absolute values of stop-ERP voltage deflections in the interval periods marked by gray areas within SSRT.
D

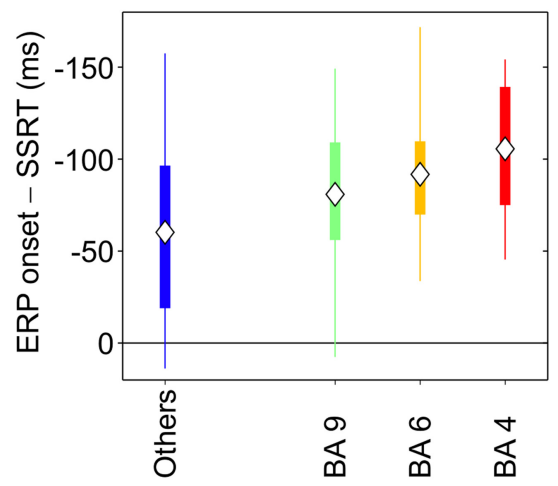

Dashed line: threshold value for selecting the subset of channels with large enough stop-ERPs used for population analyses (see Mattia et al., 2012 for details). (C) Number of channels showing large enough average stop-ERPs across five patients $(n=39)$ grouped by BA. Blue bar (others) represents those areas where channels were not selected more than twice across all patients. (D) Box plot of stop-ERP onsets measured with respect to the end of SSRT across all selected channels in all patients. Stop-ERP onset was defined as the first time that an electrode voltage was significantly different from 0 . Diamonds indicate average onset times. Tick bars indicate the first and the third quartile. Vertical lines indicate the extreme time lags in the channel group. Freely adapted from Mattia et al. (2012), with permission.
Additionally, it must be taken into account that arm movements, unlike saccades, are not ballistic movements as they can be stopped at any point along their path (De Jong et al., 1990; Scangos and Stuphorn, 2010). As a consequence, their planning can be modified even during the execution phase. This is in line with the findings of Mirabella et al. (2008), who compared RTs and movement times (MTs) of reaching movements toward visual targets executed either during an RT task (go-only task) or during a countermanding task. In both tasks subjects executed the same movements, but in the countermanding task subjects were aware that a stop-signal could randomly appear during movement preparation, indicating that the pending action should be suppressed. The awareness of the possible appearance of the stopsignal creates a conflict on all no-stop trials because, despite the 
instructions to always move as fast as possible, subjects spontaneously tend to delay movement initiation to wait for the possible occurrence of a stop-signal (e.g., Mirabella et al., 2006, 2012). This is a common proactive strategy that subjects implicitly adopt to have a greater chance of stopping their movements. However, the proactive strategy was also found to affect the MTs, which were shorter during the no-stop trials and longer during the go-only trials. Probably the increased length of RTs during the no-stop trials allowed subjects to fully process movement parameters and thus to move faster. In contrast, in go-only trials the absence of a proactive brake allows a shortening of RTs, at the cost of leaving some details of the motor program uncompleted, so that the planning must be completed during the movement. This strategy represents an optimization of costs versus benefits because shorter RTs are compensated by longer MTs and vice versa.

Interestingly, Mirabella et al. (2013) demonstrated that STN takes part in this process. In fact PD patients in which both DBS were turned on behaved similarly to healthy subjects, whereas when both DBS were off the same patients had both RTs and MTs longer in no-stop trials than in go-only trials (Figure 8). Therefore this study demonstrated the existence of a causal link between the
DBS of STN and the motor strategy exploited. Once again this evidence favors the hypothesis that STN is not involved in a single function (e.g., reactive inhibition); instead, more generally, it can compute the payoff of an ongoing action.

\section{THE MONITORING SYSTEM}

In order to learn how to make good decisions, the brain needs to compute, learn, and store the results of the outcomes that were generated by its previous decisions. To this end the ability of computing predictions about future reward and the ability of measuring discrepancies between attended and actual outcomes are fundamental. These functions are performed by a set of brain regions that collectively I will call the "monitoring system" (Figure 1). This system is composed of a set of cortical and subcortical structures that allow coding of reward expectations, detection of errors and implementation of behavioral adjustments, aiming to cope with more or less demanding context in order to optimize future choices. Therefore signals produced by the monitoring system can influence any stage of action implementation.

A key role in this set of processes is played by the ACC, located in the frontal medial wall (Figure 3). It has been known for

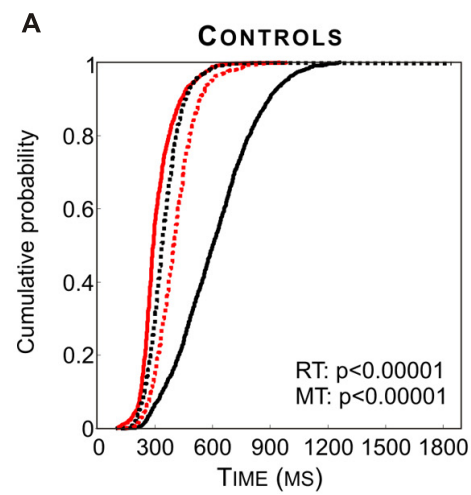

C

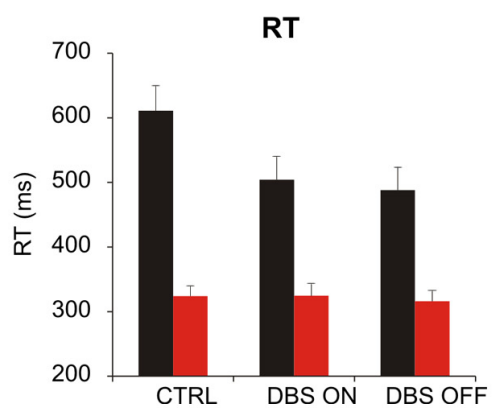

FIGURE 8 | Deep brain stimulation (DBS) of subthalamic nucleus (STN) partially restores the appropriate motor strategy according to the contexts. (A) Cumulative distribution of RTs (solid lines) and MTs (dotted lines) of healthy subjects $(n=13)$ for go-only (red lines) and no-stop (black lines) trials. (B) Cumulative distributions of RTs (solid lines) and MTs (dotted lines) of DBS patients $(n=12)$ in DBS-ON and DBF-OFF conditions for both go-only (red lines) and no-stop (black lines) trials. For
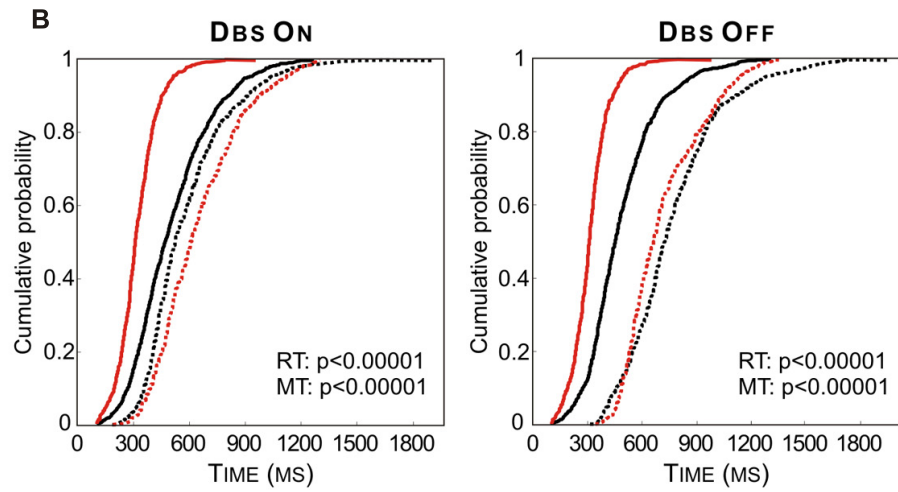

D
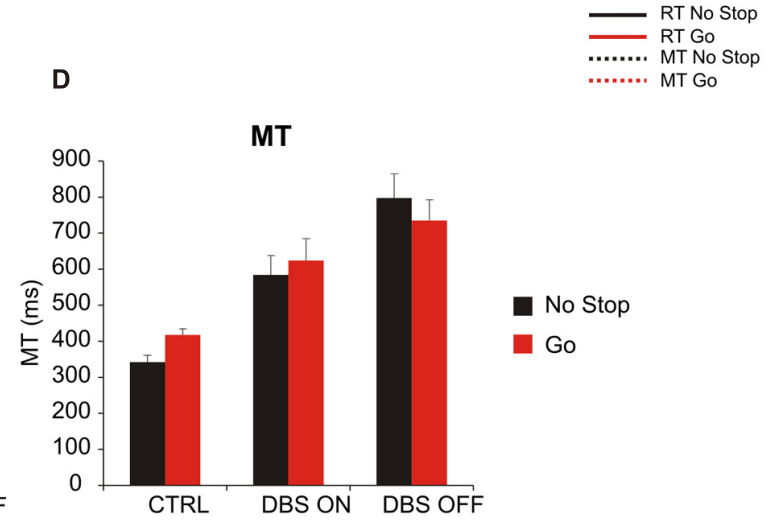

each condition the $P$-value of Kolmogorov-Smirnov test is given, both for RTs and for MTs. (C) Histograms of average RTs of no-stop and go-only trials in DBS-ON and DBF-OFF conditions. Bars represent the standard error of the mean. (D) Histograms of average MTs of no-stop and go-only trials in each DBS-ON and DBF-OFF condition. Bars represent the standard error of the mean. Reproduced from Mirabella etal. (2013), with permission from PLOS. 
a long time that this area is involved in cognitive control over behavior (e.g., Bernstein et al., 1995; Botvinick et al., 1999); however, its precise role remains considerably debated. One influential hypothesis is that the activity of ACC might represent the likelihood of obtaining or losing reward in response to particular actions. Evidence in support of this idea comes from the results of Brown and Braver (2005). They administered to human volunteers a stop-change task while recording the fMRI BOLD signal. The task, (Figure 9), required participants to make rapid leftor right-hand button-press responses according to the direction of an arrow. In $33 \%$ of cases, during the RTs, a second arrow pointing in the opposite direction was presented and the responses had to be reversed. Using a staircase algorithm, authors adjusted the time of presentation of the second arrow, hence controlling the likelihood of making mistakes (either 10 or $50 \%$ ). At the beginning of each trial, participants were informed of the error likelihood by a color cue: a blue bar indicated $50 \%$ error probability, while a white bar indicated $10 \%$ error probability. The activity of ACC was greater during the high error than during the low error conditions. This held true not only during change trials, but also during trials in which participants did not have to change the responses (Figure 9). Therefore ACC does not simply indicate response conflict (occurring when the two arrows are presented) or error occurrence; instead more generally it signals the perceived likelihood of potential errors. Crucially, Brown and Braver (2005) exploiting a neural-network model, showed that this pattern of response gradually emerged over the course of the experimental session, i.e., after individuals learned the associations between color cue and error likelihood.

Other studies point to a slightly different role for the ACC. According to these accounts, ACC would provide a continuously updated prediction of expected cognitive demand which will be used to optimize future behavior. For instance, Sheth et al. (2012) showed that ACC activity speeds up behavioral responses when cognitive demand remain stable, but, in more challenging situations, it slows down responses to allow a greater accuracy. Kennerley et al. (2006), studying the behavioral responses of monkeys before and after ACC lesions, confirmed that this region adaptively guides future behaviors, but exploiting a different mechanism. In a key experiment, monkeys were rewarded when performing a certain action (e.g., lifting a lever) until the rewarded action was changed (e.g., to get the reward it had to turn the lever). Non-lesioned monkeys had no difficult in this task, while ACClesioned monkeys following an unrewarded lift response switched to turning, but could not sustain this response on subsequent trials. Thus, the lesion compromised the ability to associate payoffs with the outcome of past actions to adaptively guide future behavior.

Whatever the exact role of ACC in monitoring behavioral performance, it appears that this capability develops through experience. It is very probable that the knowledge of past experiences is built through reinforcement-learning processes, mediated by the discharge of midbrain dopamine neurons. The discharge of these neurons measures deviations from individuals' previous reward expectations, i.e., they compute the so-called prediction errors (Schultz et al., 1997; O’Doherty et al., 2003; Bayer and Glimcher,
2005). Every time an event is better than expected, dopaminergic neurons phasically increase their discharge. In contrast, they do not change firing rate when an event occurs as predicted, while they decrease their discharge if something worse than expected takes place. Prediction errors are thought to play a key role in guiding decision-making by signaling the need to adjust future behavior, i.e., they are fundamental to learning processes (Schultz et al., 1997; Montague et al., 2004).

Dopaminergic neurons project widely to the striatum and to several regions of PFC including the ACC (Freund et al., 1984; Smith et al., 1994). The activity of ventral striatum seems to correlate with prediction error computation (Bray and O'Doherty, 2007; Hare et al., 2008) and thus it is likely to contribute to some further elaboration of action-outcome predictions. However, ACC plays a different role from ventral striatum, exploiting prediction errors as training signals to build extended action-outcome histories, that later could be exploited to adapt goal-directed behaviors (Holroyd and Coles, 2002; Brown and Braver, 2005; Kennerley et al., 2006). Given such a complex function, it is unlikely that ACC would rely solely on signals coming from dopaminergic neurons. In fact, other brain regions have been shown to produce signals promoting learning. For instance, it has been shown that neurons of the frontal pole cortex code the outcome of actions (Tsujimoto et al., 2010; for a review see Tsujimoto et al., 2011). Also, neurons in SMA and pre-SMA of monkeys have been shown to be modulated by the amount of expected reward and thus these regions might, among other things, be signaling expectation of reward too (Scangos and Stuphorn, 2010). Not all studies point to this conclusion, e.g., recently Bonini et al. (2014), by recording evoked field potentials in pharmacoresistant epileptic patients, claimed that SMA continuously assesses ongoing actions and, when an error occurs, it signals to ACC.

Whatever the exact role of each of these components, they form a system that is capable of monitoring actions, evaluating their behavioral outcomes, and learning the association between a certain environmental context and the likelihood that a certain action or strategy will lead to the desired goal. Thus, the monitoring system is the ideal candidate for implementing proactive preparation of action plans in anticipation of known task demands. Such advance preparation has been studied in the context of response inhibition, in particular when the countermanding task is employed. This is because the rules of this paradigm produce a conflict every time subjects have to execute no-stop trials (see Figure 6A). As described above, this context induces a lengthening of the RTs and at the same time a shortening of MTs with respect to situations in which the same movements have to be performed, but stop-signals are never presented (e.g., Mirabella et al., 2008, 2013).

The study of the neural underpinnings of proactive control revealed that the network of brain regions that subserve this function largely overlaps with those subserving reactive inhibition. This conclusion stems from several fMRI studies which employed a probabilistic stop task with cues indicating the likelihood of stop trial occurrence (e.g., Chikazoe et al., 2009; Zandbelt and Vink, 2010; Jahfari et al., 2012; Zandbelt et al., 2013). Some cues indicate that go-signals are never followed by a stop-signal (certain go-signals), whereas others indicate that go-signals have a 
A

\section{Low error condition}

B

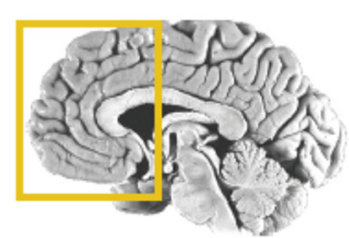

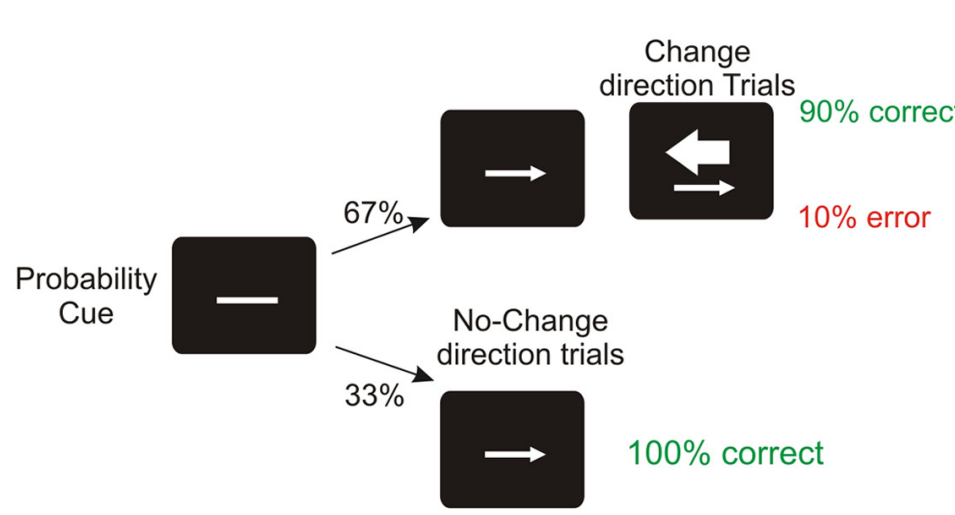

High error condition

Before learning
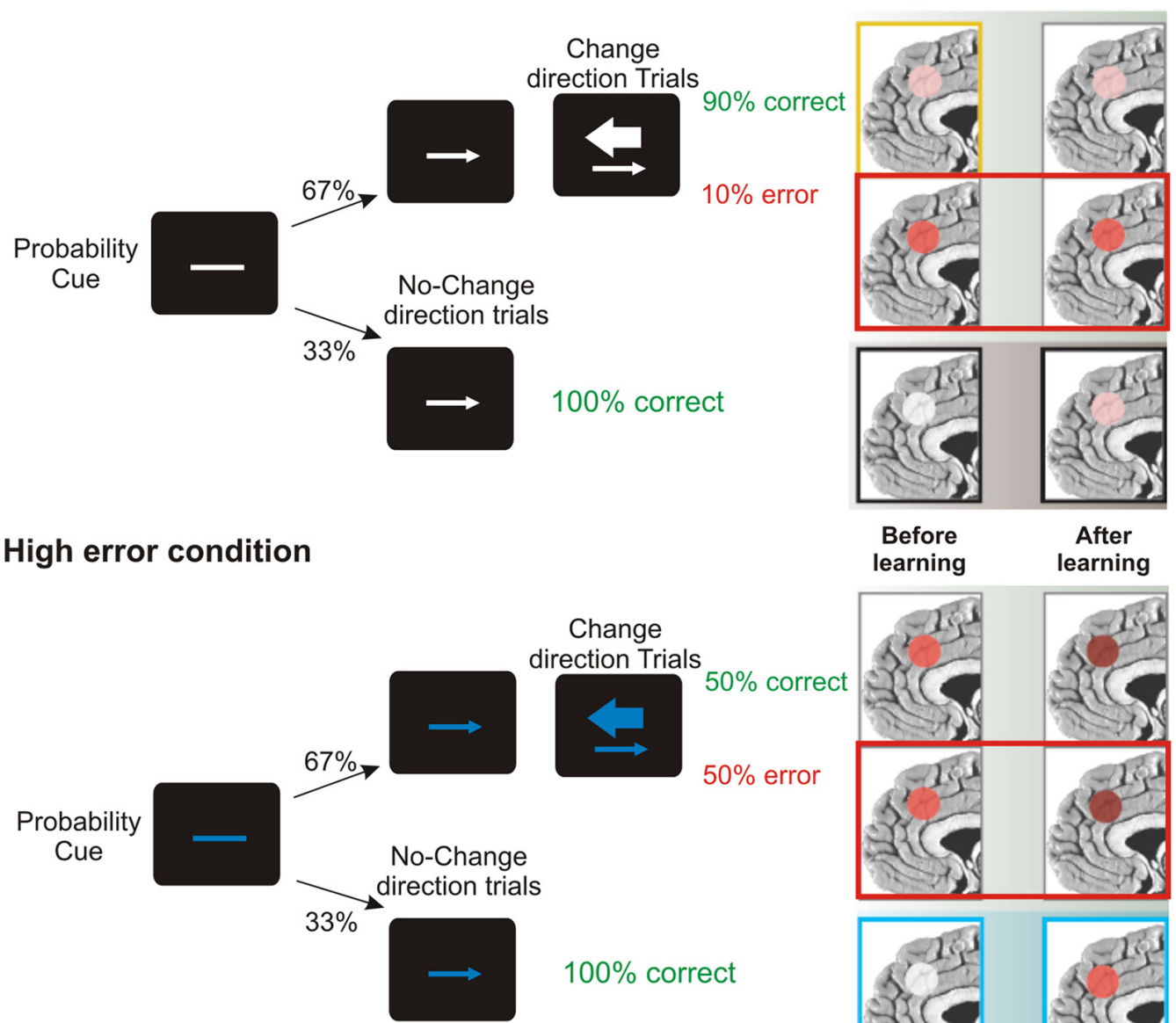

Change direction Trials
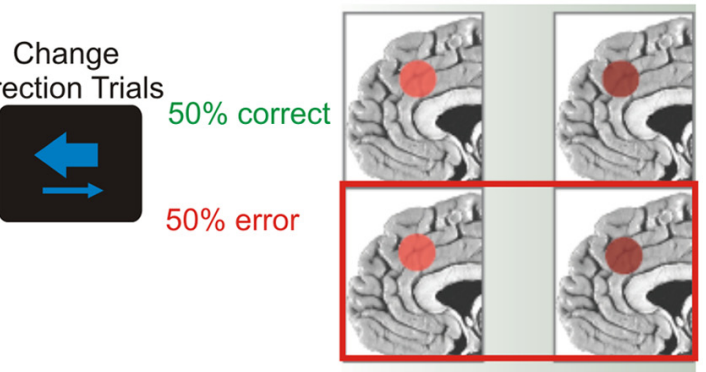

$100 \%$ correct

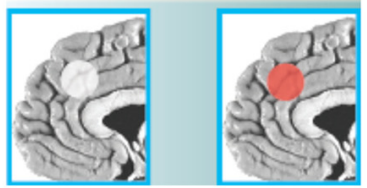

FIGURE 9 | Role of ACC in predicting error likelihood. (A) Temporal sequence of the visual displays for the change-signal task. Initially a probability cue (plain line) was displayed. The cue could be either white or blue, predicting low or high error likelihood respectively. After a delay, a go signal was presented (left or right pointing arrow) indicating the required button-press response (left or right index press for left or right pointing arrow, respectively). Randomly on $33 \%$ of the trials a change signal was displayed (a larger arrow pointing in the opposite direction with respect to that presented as go signal). To this signal, subjects had to reverse the response from that indicated by the go signal. Error rates were controlled by a staircase procedure so that in the low error condition the delay between the go signal and the change signal was kept shorter and subjects made around $90 \%$ of correct responses. In the high error condition the delay was kept longer and subjects made around 50\% correct responses. (B) Brain regions highlighted indicate the activation of ACC during the stop-change before and after learning (the greater the activation, the deeper the red color) for correct, wrong change-direction trials and for no-change trials (for the top to the bottom row) in the low and high error conditions. Interestingly, during no-change trials ACC activity increased with practice, especially in response to blue color cues, reflecting an improved ability to predict the likelihood of making an error. Freely adapted from Ridderinkhof and van den Wildenberg (2005), with permission. certain likelihood of being followed by a stop-signal (uncertain go-signals). By comparing brain activity between these conditions, it has been possible to uncover the neural substrate of advanced action preparation. Overall it emerged that when subjects expect the occurrence of a stop-signal, they proactively engage the network subserving reactive inhibition in a progressively increasing fashion according to the likelihood of stop presentation. A lower stop probability would correspond to a weaker activation of the network and to less marked slowing of responses, while when the stop is presented the activation is much stronger and the motor output is completely blocked (Aron, 2011). 
These studies did not show clear activations of either of ACC or midbrain dopaminergic neurons in the ventral tegmental area and in the substantia near pars compacta (but see Zandbelt et al., 2013). However, these might be due either to the fact that commonly a region-of-interest approach had been chosen or because of limitations of the fMRI technique. Nevertheless, the activation of other components of the monitoring system, i.e., striatum, SMA and pre-SMA, has been shown several times (Chikazoe et al., 2009; Zandbelt and Vink, 2010; Jahfari et al., 2012; Zandbelt et al., 2013). This activity might reflect the computations which underlie learning of the association between the experimental context and the likelihood of performing (or stopping) an action. Thus, these signals should drive the proactive network so as to maximize the probability of moving without missing stop trials. Further studies are needed to test this hypothesis.

\section{CONCLUDING THOUGHTS}

On the whole, it seems clear that the neural network subserving goal-directed actions is very extensive, encompassing both frontal and parietal area as well as subcortical brain structures. This does not come as a surprise, as we live in a complex and ever-changing environment, which continuously offers an enormous variety of opportunities for potential actions. Thus, our motor system is called on to perform a continuous evaluation of alternative actions that may become available, in order to decide whether to persist in a given activity or to stop it and switch to a different one. This is the hinge of our behavioral flexibility. Central to this feature are the abilities of predicting the likelihood of achieving the desired goal with a certain action or strategy, and of canceling pending actions when they are no longer valuable. These two processes operate continuously during the entire course of a movement, during its genesis, its planning, and even its execution, so that the motor output can be modulated or suppressed at any time before its execution.

These considerations are clearly at odds with the old-fashioned serial framework according to which "we sense the world, think about it, and then act upon it" (Cisek and Kalaska, 2010, p. 271). Instead, they are compatible with the idea of parallel processing. Thus, one or more goals or actions can be coded at the same time, so that alternatives can be ready for release at short notice. Importantly, the evaluation of an action can also lead to its suppression when it has already been selected. Very probably the neural machinery underling action preparation and the neural substrates underling action inhibition are simultaneously active. From what is stated above, it is not too surprising that many of the regions mediating the decisions to act appear to be involved also in decisions to refrain from acting (Coxon et al., 2006; Mirabella et al., 2011; Mattia et al., 2012; see Table 1). This leads to the main point of the present review. Many, if not all, brain regions belonging to the network which controls goal-oriented movements are involved in more stages of this process. For instance, the SMA and the pre-SMA are involved in the early "should-I-stay-or-shouldI-go" decision as they are involved in gating action affordances (e.g., Sumner et al., 2007; Ridderinkhof et al., 2011). However, they are also part of the monitoring system (see The Monitoring
System) and they contribute to the late "should-I-stay-or-shouldI-go" decision (Aron and Poldrack, 2006; Aron et al., 2007). In addition it has been shown that stimulation of SMA generates a feeling of an urge to move a particular body part, without necessarily causing any actual movement (Fried et al., 1991). The involvement of SMA in the feeling of volition has recently been confirmed by Fried et al. (2011) who recorded single-neuron activity in epileptic pharmacoresistant patients. Both PMd and M1 are involved both in the genesis of motor plans (Tanji and Evarts, 1976; Weinrich et al., 1984; Hoshi and Tanji, 2000, 2002; Churchland et al., 2006; Mattia et al., 2013; Shenoy et al., 2013) and also in their inhibition (Coxon et al., 2006; Mirabella et al., 2011; Mattia et al., 2012). Most of the network subserving reactive inhibition is also involved in proactive inhibition (see Action Execution).

Some other brain regions are involved not solely in motor controls, but also in very different tasks. The ACC has been shown to be involved in monitoring behavioral performance (see The Monitoring System), but also in pain processing (Singer et al., 2004). The role of the IFG is even more controversial as it seems to perform several different functions, some of which are lateralized (see Liakakis et al., 2011 for a review). The left IFG is implicated in both perceptive and productive aspects of language (see Uddén and Bahlmann, 2012 for a review). Some studies indicate that the right IFG plays a key role in redirecting selective attention toward unexpected stimuli (Corbetta and Shulman, 2002); others suggest that it has a specific role in motor inhibition (Aron et al., 2014) or in suppression of unwanted memories (Benoit and Anderson, 2012). In addition, the IFG of both hemispheres are involved in processing of symbolic gestures used for social non-verbal communication (Lindenberg et al., 2012), and they are regions belonging to the core of the human mirror-neuron system, i.e., of the system that is thought to allow the ability of understanding the intentions of others (for a review see Rizzolatti and Craighero, 2004). Finally, the PFC contribute to an enormous array of functions (for a review see Wise, 2008).

Given this picture, I believe that is rather hard to assign a very specific role to most of those regions when performing complex cognitive functions, such as the augmentation of adaptive behaviors. Possibly, complex cognitive functions are not performed by a unique structure. Rather, they might emerge from the coordinated activity of large-scale neuronal networks that are dynamically configured on fixed anatomical connections (von der Malsburg et al., 2010). The mechanisms underlying the dynamic coordination of neural populations are poorly understood. Nevertheless, it has been suggested that the formation of functional networks is achieved by modulating the degree of coherence among temporally structured responses of widely distributed neurons. In its turn, coherence might be modulated by rhythmic modulation of activity and synchronization among these populations (see Fries, 2005 for a review). According to this framework a given brain region can perform specific operations, e.g., the right IFG might subserve the reorienting of selective attention (Corbetta and Shulman, 2002) or the ACC might compute the likelihood of a successful action, but its functional features can be exploited in a variety of tasks. Thus the outcome of complex processes would depend not upon the activation of one or a few brain regions, but on the specific interactions of the network activated during 
the ongoing task. This hypothesis would explain why the same regions are active under many different contexts, and it might also help to understand the limited success achieved by brain-machine interfaces (BMIs; for reviews see Baranauskas, 2014; Bensmaia and Miller, 2014).

The large majority of BMI interfaces are aimed at restoring body mobility in patients suffering from motor deficits caused by brain injury, neurologic diseases and limb loss (Bensmaia and Miller, 2014). BMIs record brain signals, decode movement-related information and use these to control external devices (e.g., prosthetic limbs, wheelchairs). Although at present the BMI approach had been fruitful (e.g., Hatsopoulos and Donoghue, 2009) it has also shown several limitations. Patients have to undergo a long period of training before learning how to use the BMIs, and signals extracted from the brain are noisy and difficult to interpret so the error rate is rather high and the response speed is slow. All in all, the guidance of external devices is still far from approximating natural behaviors. This applies to both non-invasive and invasive BMIs. Non-invasive BMIs are mainly based on EEG signals recorded from the scalp. Activity recorded at the scalp level lacks selectivity; it is made up of a mix of signals coming from different cortical regions and thus the amount of useable information conveyed is likely to be too low (Baranauskas, 2014). On the other hand, invasive BMIs employ multiple electrodes surgically inserted into the brain (usually in M1 and/or the somatosensory cortex). Even though the quality of the recorded signals is definitely better, information transfer rate is still not dramatically improved (Baranauskas, 2014). The BMI approach has been shown to reproduce relatively simple behaviors such as two-dimensional center-out reaching tasks (e.g., Wolpaw and McFarland, 2004). Other much more complex tasks, e.g., those entailing suppression of pending actions (Mirabella, 2012), have almost never been tested (but see Ifft et al., 2012). The relatively low degree of success of invasive BMI might be due to the fact that, due to ethical reasons, it has been possible to capture only a very limited amount of the activity of the neural network underling goal-directed actions. However, as stated above, flexible control of behavior can be achieved only through the functional interactions of several brain regions, so it is possible that signals recorded from a limited cortical area fail to provide enough information to reproduce natural movements. To overcome these limitations, it would be necessary to find a way to record simultaneously from several key regions underlying goal-directed actions. Clearly, this is currently not feasible on humans beings, though it could be tested on non-human primates.

Before concluding, I would like to make an important remark: all processes described in the present review, i.e., genesis and suppression of goal-directed actions, do not require either intention, volition, or awareness. These computations can be performed by most animals, and surely by all primates. Not by chance, many of the data described here come from studies on non-human primates. What exactly volition or free will is and how and when it is generated from brain activity is, at best, unclear. There is a consensus around the idea originally proposed by Libet et al. (1983) that the awareness of intention precedes movements by some 100s of milliseconds. Thus, it is possible that the brain generates a action and, only subsequently, some pre-motor processes produce the subjective experience of willing to execute that action, which is perceived as being freely chosen (Hallett, 2007). To overcome the impasse of these findings, Libet (1985) proposed that because awareness of intention precedes movements, our free will should rely on the ability to withhold upcoming actions (see Mirabella, 2007; Haggard, 2008 for reviews). However, it has been shown that action suppression can be implemented unintentionally and unconsciously (see van Gaal et al., 2008). Furthermore, both monkeys (e.g., Mirabella et al., 2011) and rats (e.g., Eagle et al., 2008) can cancel pending movements. It has to be recognized that most studies on action inhibition in humans rely on externally and not on internally triggered stops (see Brass and Haggard, 2007 for an original approach), so further evidence has to be collected before venturing conclusions around the relationship between the veto power and willingness. As Roskies (2010) stated: "For now, [...], the most significant contribution neuroscience has made has been in allowing us to formulate novel questions about the nature of voluntary behavior, and in providing new ways of addressing them." I hope that the present review will also help to circumscribe what free will is not.

\section{ACKNOWLEDGMENTS}

I thank Maurizio Mattia, Leonardo Chelazzi, and Richard Ridderinkhof for valuable comments and helpful discussions.

\section{REFERENCES}

Aron, A. R. (2011). From reactive to proactive and selective control: developing a richer model for stopping inappropriate responses. Biol. Psychiatry 69, e55-e68. doi: 10.1016/j.biopsych.2010.07.024

Aron, A. R., Behrens, T. E., Smith, S., Frank, M. J., and Poldrack, R. A. (2007). Triangulating a cognitive control network using diffusion-weighted magnetic resonance imaging (MRI) and functional MRI. J. Neurosci. 27, 3743-3752. doi: 10.1523/JNEUROSCI.0519-07.2007

Aron, A. R., Fletcher, P. C., Bullmore, E. T., Sahakian, B. J., and Robbins, T. W. (2003). Stop-signal inhibition disrupted by damage to right inferior frontal gyrus in humans. Nat. Neurosci. 6, 115-116. doi: 10.1038/nn1003

Aron, A. R., and Poldrack, R. A. (2006). Cortical and subcortical contributions to stop signal response inhibition: role of the subthalamic nucleus. J. Neurosci. 26, 2424-2433. doi: 10.1523/JNEUROSCI.4682-05.2006

Aron, A. R., Robbins, T. W., and Poldrack, R. A. (2014). Inhibition and the right inferior frontal cortex: one decade on. Trends Cogn. Sci. 18, 177-185. doi: 10.1016/j.tics.2013.12.003

Band, G. P., van der Molen, M. W., and Logan, G. D. (2003). Horse-race model simulations of the stop-signal procedure. Acta Psychol. (Amst.) 112, 105-142. doi: 10.1016/S0001-6918(02)00079-3

Baranauskas, G. (2014). What limits the performance of current invasive brain machine interfaces? Front. Syst. Neurosci. 8:68. doi: 10.3389/fnsys.2014. 00068

Barraclough, D. J., Conroy, M. L., and Lee, D. (2004). Prefrontal cortex and decision making in a mixed-strategy game. Nat. Neurosci. 7, 404-410. doi: 10.1038/n n1209

Bayer, H. M., and Glimcher, P. W. (2005). Midbrain dopamine neurons encode a quantitative reward prediction error signal. Neuron 47, 129-141. doi: 10.1016/j.neuron.2005.05.020

Benoit, R. G., and Anderson, M. C. (2012). Opposing mechanisms support the voluntary forgetting of unwanted memories. Neuron 76, 450-460. doi: 10.1016/j.neuron.2012.07.025

Bensmaia, S. J., and Miller, L. E. (2014). Restoring sensorimotor function through intracortical interfaces: progress and looming challenges. Nat. Rev. Neurosci. 15, 313-325. doi: 10.1038/nrn3724

Bernstein, P. S., Scheffers, M. K., and Coles, M. G. (1995). "Where did I go wrong?" A psychophysiological analysis of error detection. J. Exp. Psychol. Hum. Percept. Perform. 21, 1312-1322. doi: 10.1037/0096-1523.21.6.1312 
Biran, I., and Chatterjee, A. (2004). Alien hand syndrome. Arch. Neurol. 61, 292-294. doi: 10.1001/archneur.61.2.292

Boccardi, E., Della Sala, S., Motto, C., and Spinnler, H. (2002). Utilisation behaviour consequent to bilateral SMA softening. Cortex 38, 289-308. doi: 10.1016/S00109452(08)70661-0

Bonanni, L., Thomas, A., and Onofrj, M. (2010). Paradoxical kinesia in parkinsonian patients surviving earthquake. Mov. Disord. 25, 1302-1304. doi: 10.1002/mds. 23075

Bonini, F., Burle, B., Liégeois-Chauvel, C., Régis, J., Chauvel, P., and Vidal, F. (2014). Action monitoring and medial frontal cortex: leading role of supplementary motor area. Science 343, 888-891. doi: 10.1126/science. 1247412

Botvinick, M., Nystrom, L. E., Fissell, K., Carter, C. S., and Cohen, J. D. (1999). Conflict monitoring versus selection-for-action in anterior cingulate cortex. Nature 402, 179-181. doi: 10.1038/46035

Boucher, L., Palmeri, T. J., Logan, G. D., and Schall, J. D. (2007). Inhibitory control in mind and brain: an interactive race model of countermanding saccades. Psychol. Rev. 114, 376-397. doi: 10.1037/0033-295X.114.2.376

Brass, M., and Haggard, P. (2007). To do or not to do: the neural signature of self-control. J. Neurosci. 27, 9141-9145. doi: 10.1523/JNEUROSCI.092407.2007

Bray, S., and O’Doherty, J. (2007). Neural coding of reward-prediction error signals during classical conditioning with attractive faces. J. Neurophysiol. 97, 3036-3045. doi: 10.1152/jn.01211.2006

Brown, J. W., and Braver, T. S. (2005). Learned predictions of error likelihood in the anterior cingulate cortex. Science 307, 1118-1121. doi: 10.1126/science.11 05783

Calton, J. L., Dickinson, A. R., and Snyder, L. H. (2002). Non-spatial, motorspecific activation in posterior parietal cortex. Nat. Neurosci. 5, 580-588. doi: 10.1038/nn0602-862

Camille, N., Coricelli, G., Sallet, J., Pradat-Diehl, P., Duhamel, J. R., and Sirigu, A. (2004). The involvement of the orbitofrontal cortex in the experience of regret. Science 304, 1167-1170. doi: 10.1126/science. 1094550

Chambers, C. D., Bellgrove, M. A., Stokes, M. G., Henderson, T. R., Garavan, H., Robertson, I. H., et al. (2006). Executive "brake failure" following deactivation of human frontal lobe. J. Cogn. Neurosci. 18, 444-455. doi: 10.1162/089892906775990606

Chen, X., Scangos, K. W., and Stuphorn, V. (2010). Supplementary motor area exerts proactive and reactive control of arm movements. J. Neurosci. 30, 14657-14675. doi: 10.1523/JNEUROSCI.2669-10.2010

Chikazoe, J., Jimura, K., Hirose, S., Yamashita, K., Miyashita, Y., and Konishi, S. (2009). Preparation to inhibit a response complements response inhibition during performance of a stop-signal task. J. Neurosci. 29, 15870-15877. doi: 10.1523/JNEUROSCI.3645-09.2009

Churchland, M. M., and Shenoy, K. V. (2007). Delay of movement caused by disruption of cortical preparatory activity. J. Neurophysiol. 97, 348-359. doi: 10.1152/jn.00808.2006

Churchland, M. M., Yu, B. M., Ryu, S. I., Santhanam, G., and Shenoy, K. V. (2006). Neural variability in premotor cortex provides a signature of motor preparation. J. Neurosci. 26, 3697-3712. doi: 10.1523/JNEUROSCI.376205.2006

Cisek, P. (2006). Integrated neural processes for defining potential actions and deciding between them: a computational model. J. Neurosci. 26, 9761-9770. doi: 10.1523/JNEUROSCI.5605-05.2006

Cisek, P., and Kalaska, J. F. (2005). Neural correlates of reaching decisions in dorsal premotor cortex: specification of multiple direction choices and final selection of action. Neuron 45, 801-814. doi: 10.1016/j.neuron.2005.01.027

Cisek, P., and Kalaska, J. F. (2010). Neural mechanisms for interacting with a world full of action choices. Annu. Rev. Neurosci. 33, 269-298. doi: 10.1146/annurev.neuro.051508.135409

Corbetta, M., and Shulman, G. L. (2002). Control of goal-directed and stimulusdriven attention in the brain. Nat. Rev. Neurosci. 3, 201-215. doi: 10.1038/n rn755

Coxon, J. P., Stinear, C. M., and Byblow, W. D. (2006). Intracortical inhibition during volitional inhibition of prepared action. J. Neurophysiol. 95, 3371-3383. doi: 10.1152/jn.01334.2005

Cui, H., and Andersen, R. A. (2007). Posterior parietal cortex encodes autonomously selected motor plans. Neuron 56, 552-559. doi: 10.1016/j.neuron.2007. 09.031
Damasio, A. R. (1994). Descartes' Error: Emotion, Reason, and the Human Brain. New York: Putman.

De Jong, R., Coles, M. G., Logan, G. D., and Gratton, G. (1990). In search of the point of no return: the control of response processes. J. Exp. Psychol. Hum. Percept. Perform. 16, 164-182. doi: 10.1037/0096-1523.16.1.164

Della Sala, S., Marchetti, C., and Spinnler, H. (1991). Right-sided anarchic (alien) hand: a longitudinal study. Neuropsychologia 29, 1113-1127. doi: 10.1016/00283932(91)90081-I

Eagle, D. M., Baunez, C., Hutcheson, D. M., Lehmann, O., Shah, A. P., and Robbins, T. W. (2008). Stop-signal reaction-time task performance: role of prefrontal cortex and subthalamic nucleus. Cereb. Cortex 18, 178-188. doi: 10.1093/cercor/b hm044

Fellows, L. K., and Farah, M. J. (2007). The role of ventromedial prefrontal cortex in decision making: judgment under uncertainty or judgment per se? Cereb. Cortex 17, 2669-2674. doi: 10.1093/cercor/bhl176

Forstmann, B. U., van den Wildenberg, W. P. M., and Ridderinkhof, K. R. (2008) Neural mechanisms, temporal dynamics, and individual differences in interference control. J. Cogn. Neurosci. 20, 1854-1865. doi: 10.1162/jocn.2008. 20122

Freedman, D. J., Riesenhuber, M., Poggio, T., and Miller, E. K. (2002). Visual categorization and the primate prefrontal cortex: neurophysiology and behavior. J. Neurophysiol. 88, 929-941.

Freund, T. F., Powell, J. F., and Smith, A. D. (1984). Tyrosine hydroxylaseimmunoreactive boutons in synaptic contact with identified striatonigral neurons, with particular reference to dendritic spines. Neuroscience 13, 1189-1215. doi: 10.1016/0306-4522(84)90294-X

Fried, I., Katz, A., McCarthy, G., Sass, K. J., Williamson, P., Spencer, S. S., et al. (1991). Functional organization of human supplementary motor cortex studied by electrical stimulation. J. Neurosci. 11, 3656-3666.

Fried, I., Mukamel, R., and Kreiman, G. (2011). Internally generated preactivation of single neurons in human medial frontal cortex predicts volition Neuron 69, 548-562. doi: 10.1016/j.neuron.2010.11.045

Fries, P. (2005). A mechanism for cognitive dynamics: neuronal communication through neuronal coherence. Trends Cogn. Sci. 9, 474-480. doi: 10.1016/j.tics.2005.08.011

Funahashi, S., Bruce, C. J., and Goldman-Rakic, P. S. (1989). Mnemonic coding of visual space in the monkey's dorsolateral prefrontal cortex. J. Neurophysiol. 61, 331-349.

Genovesio, A., Brasted, P. J., Mitz, A. R., and Wise, S. P. (2005). Prefrontal cortex activity related to abstract response strategies. Neuron 47, 307-320. doi: 10.1016/j.neuron.2005.06.006

Genovesio, A., Brasted, P. J., and Wise, S. P. (2006). Representation of future and previous spatial goals by separate neural populations in prefrontal cortex. J. Neurosci. 26, 7305-7316. doi: 10.1523/JNEUROSCI.0699-0 6.2006

Genovesio, A., Tsujimoto, S., and Wise, S. P. (2012). Encoding goals but not abstract magnitude in the primate prefrontal cortex. Neuron 74, 656-662. doi: 10.1016/j.neuron.2012.02.023

Genovesio, A., Wise, S. P., and Passingham, R. E. (2014). Prefrontal-parietal function: from foraging to foresight. Trends Cogn. Sci. 18, 72-81. doi: 10.1016/j.tics.2013.11.007

Georgopoulos, A. P., Kalaska, J. F., Caminiti, R., and Massey, J. T. (1982). On the relations between the direction of two-dimensional arm movements and cell discharge in primate motor cortex. J. Neurosci. 2, 1527-1537.

Gibson, J. J. (1979). The Ecological Approach to Visual Perception. Boston, MA: Houghton-Mifflin.

Glickstein, M., and Stein, J. (1991). Paradoxical movement in Parkinson's disease. Trends Neurosci. 14, 480-482. doi: 10.1016/0166-2236(91)9 0055-Y

Glimcher, P. W., Dorris, M. C., and Bayer, H. M. (2005). Physiological utility theory and the neuroeconomics of choice. Games Econ. Behav. 52, 213-256. doi: 10.1016/j.geb.2004.06.011

Grezes, J., and Decety, J. (2002). Does visual perception of object afford action? Evidence from a neuroimaging study. Neuropsychologia 40, 212-222. doi: 10.1016/S0028-3932(01)00089-6

Habib, M. (2004). Athymhormia and disorders of motivation in basal ganglia disease. J. Neuropsychiatry Clin. Neurosci. 16, 509-524. doi: 10.1176/appi.neuropsych.16.4.509 
Haggard, P. (2008). Human volition: towards a neuroscience of will. Nat. Rev. Neurosci. 9, 934-946. doi: 10.1038/nrn2497

Hallett, M. (2007). Volitional control of movement: the physiology of free will. Clin. Neurophysiol. 118, 1179-1192. doi: 10.1016/j.clinph.2007.03.019

Hanes, D. P., and Schall, J. D. (1996). Neural control of voluntary movement initiation. Science 274, 427-430. doi: 10.1126/science.274.528 6.427

Hare, T. A., O’Doherty, J., Camerer, C. F., Schultz, W., and Rangel, A. (2008) Dissociating the role of the orbitofrontal cortex and the striatum in the computation of goal values and prediction errors. J. Neurosci. 28, 5623-5630. doi: 10.1523/JNEUROSCI.1309-08.2008

Hatsopoulos, N. G., and Donoghue, J. P. (2009). The science of neural interface systems. Annu. Rev. Neurosci. 32, 249-266. doi: 10.1146/annurev.neuro.051508.135241

Holroyd, C. B., and Coles, M. G. (2002). The neural basis of human error processing: reinforcement learning, dopamine, and the error-related negativity. Psychol. Rev. 109, 679-709. doi: 10.1037/0033-295X.109.4.679

Hoshi, E., Shima, K., and Tanji, J. (2000). Neuronal activity in the primate prefrontal cortex in the process of motor selection based on two behavioral rules. J. Neurophysiol. 83, 2355-2373.

Hoshi, E., and Tanji, J. (2000). Integration of target and body-part information in the premotor cortex when planning action. Nature 408, 466-470. doi: $10.1038 / 35044075$

Hoshi, E., and Tanji, J. (2002). Contrasting neuronal activity in the dorsal and ventral premotor areas during preparation to reach. J. Neurophysiol. 87, 1123-1128.

Humphreys, G. W., and Riddoch, M. J. (2000). One more cup of coffee for the road: object-action assemblies, response blocking and response capture after frontal lobe damage. Exp. Brain Res. 133, 81-93. doi: 10.1007/s0022100 00403

Ifft, P. J., Lebedev, M. A., and Nicolelis, M. A. (2012). Reprogramming movements: extraction of motor intentions from cortical ensemble activity when movement goals change. Front. Neuroeng. 5:16. doi: 10.3389/fneng.2012. 00016

Jahfari, S., Stinear, C. M., Claffey, M., Verbruggen, F., and Aron, A. R. (2010). Responding with restraint: what are the neurocognitive mechanisms? J. Cogn. Neurosci. 22, 1479-1492. doi: 10.1162/jocn.2009.21307

Jahfari, S., Verbruggen, F., Frank, M. J., Waldorp, L. J., Colzato, L., Ridderinkhof, K. R., et al. (2012). How preparation changes the need for top-down control of the basal ganglia when inhibiting premature actions. J. Neurosci. 32, 10870-10878. doi: 10.1523/JNEUROSCI.0902-12.2012

Kennerley, S. W., Walton, M. E., Behrens, T. E. J., Buckley, M. J., and Rushworth, M. F. S. (2006). Optimal decision making and the anterior cingulate cortex. Nat. Neurosci. 9, 940-947. doi: 10.1038/nn1724

Klaes, C., Westendorff, S., Chakrabarti, S., and Gail, A. (2011). Choosing goals, not rules: deciding among rule-based action plans. Neuron 70, 536-548. doi 10.1016/j.neuron.2011.02.053

Koenigs, M., and Tranel, D. (2007). Irrational economic decision-making after ventromedial prefrontal damage: evidence from the Ultimatum Game. J. Neurosci. 27, 951-956. doi: 10.1523/JNEUROSCI.4606-06.2007

Lebedev, M. A., Messinger, A., Kralik, J. D., and Wise, S. P. (2004). Representation of attended versus remembered locations in prefrontal cortex. PLoS Biol. 2:e365. doi: 10.1371/journal.pbio.0020365

Lebedev, M. A., and Wise, S. P. (2001). Tuning for the orientation of spatial attention in dorsal premotor cortex. Eur. J. Neurosci. 13, 1002-1008. doi: 10.1046/j.0953816x.2001.01457.x

Li, C. S., Yan, P., Sinha, R., and Lee, T. W. (2008). Subcortical processes of motor response inhibition during a stop signal task. Neuroimage 41, 1352-1363. doi: 10.1016/j.neuroimage.2008.04.023

Liakakis, G., Nickel, J., and Seitz, R. J. (2011). Diversity of the inferior frontal gyrus - a meta-analysis of neuroimaging studies. Behav. Brain Res. 225, 341-347. doi: 10.1016/j.bbr.2011.06.022

Libet, B. (1985). Unconscious cerebral initiative and the role of conscious will in voluntary action. Behav. Brain Sci. 8, 529-566. doi: 10.1017/S0140525X00044903

Libet, B., Gleason, C. A., Wright, E. W., and Pearl, D. K. (1983). Time of conscious intention to act in relation to onset of cerebral activity (readiness-potential). The unconscious initiation of a freely voluntary act. Brain 106, 623-642. doi: 10.1093/brain/106.3.623
Lindenberg, R., Uhlig, M., Scherfeld, D., Schlaug, G., and Seitz, R. J. (2012). Communication with emblematic gestures: shared and distinct neural correlates of expression and reception. Hum. Brain Mapp. 33, 812-823. doi: 10.1002/hbm. 21258

Logan, G. D., and Cowan, W. B. (1984). On the ability to inhibit thought and action: a theory of an act of control. Psychol. Rev. 91, 295-327. doi: 10.1037/0033295X.91.3.295

Logan, G. D., and Irwin, D. E. (2000). Don't look! Don't touch! Inhibitory control of eye and hand movements. Psychon. Bull. Rev. 7, 107-112. doi: 10.3758/BF03210728

Loukas, C., and Brown, P. (2004). Online prediction of self-paced hand-movements from subthalamic activity using neural networks in Parkinson's disease. J. Neurosci. Methods 137, 193-205. doi: 10.1016/j.jneumeth.2004.02.017

Mattia, M., Pani, P., Mirabella, G., Costa, S., Del Giudice, P., and Ferraina, S. (2013). Heterogeneous attractor cell assemblies for motor planning in premotor cortex. J. Neurosci. 33, 11155-11168. doi: 10.1523/JNEUROSCI.4664-12.2013

Mattia, M., Spadacenta, S., Pavone, L., Quarato, P., Esposito, V., Sparano, A., et al. (2012). Stop-event-related potentials from intracranial electrodes reveal a key role of premotor and motor cortices in stopping ongoing movements. Front. Neuroeng. 5:12. doi: 10.3389/fneng.2012.00012

Mazzoni, P., Hristova, A., and Krakauer, J. W. (2007). Why don't we move faster? Parkinson's disease, movement vigor, and implicit motivation. J. Neurosci. 27, 7105-7116. doi: 10.1523/JNEUROSCI.0264-07.2007

Milner, D., and Goodale, M. (1995). The Visual Brain in Action. Oxford: Oxford University press.

Mirabella, G. (2007). Endogenous Inhibition and the neural basis of "free won't." J. Neurosci. 27, 13919-13920. doi: 10.1523/JNEUROSCI.4943-07.2007

Mirabella, G. (2012). Volitional inhibition and brain-machine interfaces: a mandatory wedding. Front. Neuroeng. 5:20. doi: 10.3389/fneng.2012. 00020

Mirabella, G., Bertini, G., Samengo, I., Kilavik, B. E., Frilli, D., Della Libera, C., et al. (2007). Neurons in area V4 of the macaque translate attended features into behaviorally relevant categories. Neuron 54, 303-318. doi: 10.1016/j.neuron.2007.04.007

Mirabella, G., Iaconelli, S., Modugno, N., Giannini, G., Lena, F., and Cantore, G. (2013). Stimulation of subthalamic nuclei restores a near normal planning strategy in Parkinson's patients. PLoS ONE 8:e62793. doi: 10.1371/journal.pone.0062793

Mirabella, G., Iaconelli, S., Romanelli, P., Modugno, N., Lena, F., Manfredi, M., et al. (2012). Deep brain stimulation of subthalamic nuclei affects arm response inhibition in Parkinson's patients. Cereb. Cortex 22, 1124-1132. doi: 10.1093/cercor/bhr187

Mirabella, G., Pani, P., and Ferraina, S. (2008). Context influences on the preparation and execution of reaching movements. Cogn. Neuropsychol. 25, 996-1010. doi: 10.1080/02643290802003216

Mirabella, G., Pani, P., and Ferraina, S. (2009). The presence of visual gap affects the duration of stopping process. Exp. Brain Res. 192, 199-209. doi: 10.1007/s00221008-1570-y

Mirabella, G., Pani, P., and Ferraina, S. (2011). Neural correlates of cognitive control of reaching movements in the dorsal premotor cortex of rhesus monkeys. J. Neurophysiol. 106, 1454-1466. doi: 10.1152/jn.00995.2010

Mirabella, G., Pani, P., Paré, M., and Ferraina, S. (2006). Inhibitory control of reaching movements in humans. Exp. Brain Res. 174, 240-255. doi: 10.1007/s00221-006-0456-0

Montague, P. R., Hyman, S. E., and Cohen, J. D. (2004). Computational roles for dopamine in behavioural control. Nature 431, 760-767. doi: 10.1038/nature03015

Murata, A., Fadiga, L., Fogassi, L., Gallese, V., Raos, V., and Rizzolatti, G. (1997). Object representation in the ventral premotor cortex (area F5) of the monkey. J. Neurophysiol. 78, 2226-2230.

O'Doherty, J. P., Dayan, P., Friston, K., Critchley, H., and Dolan, R. J. (2003). Temporal difference models and reward-related learning in the human brain. Neuron 38, 329-337. doi: 10.1016/S0896-6273(03)00169-7

Padoa-Schioppa, C., and Assad, J. A. (2006). Neurons in the orbitofrontal cortex encode economic value. Nature 441, 223-226. doi: 10.1038/nature04676

Padoa-Schioppa, C., and Assad, J. A. (2008). The representation of economic value in the orbitofrontal cortex is invariant for changes of menu. Nat. Neurosci. 11, 95-102. doi: $10.1038 / \mathrm{nn} 2020$ 
Paradiso, G., Saint-Cyr, J. A., Lozano, A. M., Lang, A. E., and Chen, R. (2003). Involvement of the human subthalamic nucleus in movement preparation. Neurology 61, 1538-1545. doi: 10.1212/01.WNL.0000096021.28967.57

Petrides, M., and Pandya, D. N. (1999). Dorsolateral prefrontal cortex: comparative cytoarchitectonic analysis in the human and the macaque brain and corticocortical connection patterns. Eur. J. Neurosci. 11, 1011-1136. doi: 10.1046/j.1460-9568.1999.00518.x

Petrides, M., and Pandya, D. N. (2002). Comparative cytoarchitectonic analysis of the human and the macaque ventrolateral prefrontal cortex and corticocortical connection patterns in the monkey. Eur. J. Neurosci. 16, 291-310. doi: 10.1046/j.1460-9568.2001.02090.x

Prut, Y., and Fetz, E. E. (1999). Primate spinal interneurons show pre-movement instructed delay activity. Nature 401, 590-594. doi: 10.1038/44145

Rangel, A., Camerer, C., and Montague, P. R. (2008). A framework for studying the neurobiology of value-based decision making. Nat. Rev. Neurosci. 9, 545-556. doi: $10.1038 / \mathrm{nrn} 2357$

Reynolds, J. H., and Chelazzi, L. (2004). Attentional modulation of visual processing. Annu. Rev. Neurosci. 27, 611-647. doi: 10.1146/annurev.neuro.26.041002.1 31039

Ridderinkhof, K. R., Forstmann, B. U., Wylie, S. A., Burle, B., and van den Wildenberg, W. P. M. (2011). Neurocognitive mechanisms of action control: resisting the call of the sirens. Cogn. Sci. 2, 174-192. doi: 10.1002/wcs.99

Ridderinkhof, K. R., and van den Wildenberg, W. P. (2005). Adaptive coding. Science 307, 1059-1060. doi: 10.1126/science.1109837

Ridderinkhof, K. R., van den Wildenberg, W. P., Segalowitz, S. J., and Carter, C. S. (2004). Neurocognitive mechanisms of cognitive control: the role of prefrontal cortex in action selection, response inhibition, performance monitoring, and reward-based learning. Brain Cogn. 56, 129-140. doi 10.1016/j.bandc.2004.09.016

Riehle, A., and Requin, J. (1989). Monkey primary motor and premotor cortex: single-cell activity related to prior information about direction and extent of an intended movement. J. Neurophysiol. 61, 534-549.

Rizzolatti, G., and Craighero, L. (2004). The mirror-neuron system. Annu. Rev. Neurosci. 27, 169-192. doi: 10.1146/annurev.neuro.27.070203.144230

Rizzolatti, G., and Luppino, G. (2001). The cortical motor system. Neuron 31 889-901. doi: 10.1016/S0896-6273(01)00423-8

Roskies, A. L. (2010). How does neuroscience affect our conception of volition? Annu. Rev. Neurosci. 33, 109-130. doi: 10.1146/annurev-neuro-060909-153151

Scangos, K. W., and Stuphorn, V. (2010). Medial frontal cortex motivates but does not control movement initiation in the countermanding task. J. Neurosci. 30, 1968-1982. doi: 10.1523/JNEUROSCI.4509-09.2010

Schall, J. D., and Godlove, D. C. (2012). Current advances and pressing problems in studies of stopping. Curr. Opin. Neurobiol. 22, 1012-1021. doi: 10.1016/j.conb.2012.06.002

Scherberger, H., and Andersen, R. A. (2007). Target selection signals for arm reaching in the posterior parietal cortex. J. Neurosci. 27, 2001-2012. doi 10.1523/JNEUROSCI.4274-06.2007

Schlesinger, I., Erikh, I., and Yarnitsky, D. (2007). Paradoxical kinesia at war. Mov. Disord. 22, 2394-2397. doi: 10.1002/mds.21739

Schmidt, L., d'Arc, B. F., Lafargue, G., Galanaud, D., Czernecki, V., Grabli, D., et al. (2008). Disconnecting force from money: effects of basal ganglia damage on incentive motivation. Brain 131, 1303-1310. doi: 10.1093/brain/awn045

Schultz, W., Dayan, P., and Montague, P. R. (1997). A neural substrate of prediction and reward. Science 275, 1593-1599. doi: 10.1126/science.275.5306.1593

Shenoy, K. V., Sahani, M., and Churchland, M. M. (2013). Cortical control of arm movements: a dynamical systems perspective. Annu. Rev. Neurosci. 36, 337-359. doi: 10.1146/annurev-neuro-062111-150509

Sheth, S. A., Mian, M. K., Patel, S. R., Asaad, W. F., Williams, Z. M., Dougherty, D. D., et al. (2012). Human dorsal anterior cingulate cortex neurons mediate ongoing behavioural adaptation. Nature 488, 218-221. doi: 10.1038/nature11239

Singer, T., Seymour, B., O’Doherty, J., Kaube, H., Dolan, R. J., and Frith, C. D. (2004). Empathy for pain involves the affective but not sensory components of pain. Science 303, 1157-1162. doi: 10.1126/science.1093535

Smith, P. L., and Ratcliff, R. (2004). Psychology and neurobiology of simple decisions. Trends Neurosci. 27, 161-168. doi: 10.1016/j.tins.2004. 01.006

Smith, Y., Bennett, B. D., Bolam, J. P., Parent, A., and Sadikot, A. F. (1994). Synaptic relationships between dopaminergic afferents and cortical or thalamic input in the sensorimotor territory of the striatum in monkey. J. Comp. Neurol. 344, 1-19. doi: 10.1002/cne.903440102

Stevenson, S. A., Elsley, J. K., and Corneil, B. D. (2009). A "gap effect" on stop signal reaction times in a human saccadic countermanding task. J. Neurophysiol. 101, 580-590. doi: 10.1152/jn.90891.2008

Sumner, P., Nachev, P., Morris, P., Jackson, S. R., Kennard, C., and Husain, M. (2007). Human medial frontal cortex mediates unconscious inhibition of voluntary action. Neuron 54, 697-711. doi: 10.1016/j.neuron.2007. 05.016

Swann, N., Poizner, H., Houser, M., Gould, S., Greenhouse, I., Cai, W., et al. (2011). Deep brain stimulation of the subthalamic nucleus alters the cortical profile of response inhibition in the beta frequency band: a scalp EEG study in Parkinson's disease. J. Neurosci. 31, 5721-5729. doi: 10.1523/JNEUROSCI.613510.2011

Swann, N., Tandon, N., Canolty, R., Ellmore, T. M., McEvoy, L. K., Dreyer, S., et al. (2009). Intracranial EEG reveals a time-and frequency-specific role for the right inferior frontal gyrus and primary motor cortex in stopping initiated responses. J. Neurosci. 29, 12675-12685. doi: 10.1523/JNEUROSCI.335909.2009

Tanji, J., and Evarts, E. V. (1976). Anticipatory activity of motor cortex neurons in relation to direction of an intended movement. J. Neurophysiol. 39, 1062-1068.

Tsujimoto, S., Genovesio, A., and Wise, S. P. (2010). Evaluating self-generated decisions in frontal pole cortex of monkeys. Nat. Neurosci. 13, 120-126. doi: $10.1038 / \mathrm{nn} .2453$

Tsujimoto, S., Genovesio, A., and Wise, S. P. (2011). Frontal pole cortex: encoding ends at the end of the endbrain. Trends Cogn. Sci. 15, 169-176. doi: 10.1016/j.tics.2011.02.001

Turner, R. S., and Desmurget, M. (2010). Basal ganglia contributions to motor control: a vigorous tutor. Curr. Opin. Neurobiol. 20, 704-716. doi: 10.1016/j.conb.2010.08.022

Uddén, J., and Bahlmann, J. (2012). A rostro-caudal gradient of structured sequence processing in the left inferior frontal gyrus. Philos. Trans. R. Soc. Lond. B Biol. Sci. 367, 2023-2032. doi: 10.1098/rstb.2012.0009

Van den Wildenberg, W. P., van Boxtel, G. J., van der Molen, M. W., Bosch, D. A., Speelman, J. D., and Brunia, C. H. (2006). Stimulation of the subthalamic region facilitates the selection and inhibition of motor responses in Parkinson's disease. J. Cogn. Neurosci. 18, 626-636. doi: 10.1162/jocn.2006.18.4.626

van Gaal, S., Ridderinkhof, K. R., Fahrenfort, J. J., Scholte, H. S., and Lamme, V. A. (2008). Frontal cortex mediates unconsciously triggered inhibitory control. J. Neurosci. 28, 8053-8062. doi: 10.1523/JNEUROSCI.1278-08.2008

van Gaal, S., Scholte, H. S., Lamme, V. A., Fahrenfort, J. J., and Ridderinkhof, K. R. (2011). Pre-SMA graymatter density predicts individual differences in action selection in the face of conscious and unconscious response conflict J. Cogn. Neurosci. 23, 382-390. doi: 10.1162/jocn.2010.21444

Verbruggen, F., and Logan, G. D. (2009). Proactive adjustments of response strategies in the stop-signal paradigm. J. Exp. Psychol. Hum. Percept. Perform. 35, 835-854. doi: $10.1037 / \mathrm{a} 0012726$

von der Malsburg, C., Phillips, W. A., and Singer, W. (2010). Dynamic Coordination in the Brain: From Neurons to Mind. Cambridge, MA: The MIT Press. doi: 10.7551/mitpress/9780262014717.001.0001

Wallis, J. D. (2007). Orbitofrontal cortex and its contribution to decisionmaking. Annu. Rev. Neurosci. 30, 31-56. doi: 10.1146/annurev.neuro.30.051606.0 94334

Weinrich, M., Wise, S. P., and Mauritz, K. H. (1984). A neurophysiological study of the premotor cortex in the rhesus monkey. Brain 107, 385-414. doi: 10.1093/brain/107.2.385

Wise, S. P. (2008). Forward frontal fields: phylogeny and fundamental function. Trends Neurosci. 31, 599-608. doi: 10.1016/j.tins.2008.08.008

Wise, S. P., Weinrich, M., and Mauritz, K. H. (1983). Motor aspects of cue-related neuronal activity in premotor cortex of the rhesus monkey. Brain Res. 260, 301305. doi: 10.1016/0006-8993(83)90685-6

Wolpaw, J. R., and McFarland, D. J. (2004). Control of a two-dimensional movement signal by a noninvasive brain-computer interface in humans. Proc. Natl. Acad. Sci. U.S.A. 101, 17849-17854. doi: 10.1073/pnas.0403504101

Wolpert, D. M., and Miall, R. C. (1996). Forward models for physiological motor control. Neural Netw. 9, 1265-1279. doi: 10.1016/S0893-6080(96)0 0035-4 
Zandbelt, B. B., Bloemendaal, M., Neggers, S. F., Kahn, R. S., and Vink, M. (2013). Expectations and violations: delineating the neural network of proactive inhibitory control. Hum. Brain Mapp. 34, 2015-2024. doi: 10.1002/hbm.22047

Zandbelt, B. B., and Vink, M. (2010). On the role of the striatum in response inhibition. PLoS ONE 5:e13848. doi: 10.1371/journal.pone.0013848

Zheng, D., Oka, T., Bokura, H., and Yamaguchi, S. (2008). The key locus of common response inhibition network for nogo and stop signals. J. Cogn. Neurosci. 20, 1-9. doi: 10.1162/jocn.2008.20100

Conflict of Interest Statement: The author declares that the research was conducted in the absence of any commercial or financial relationships that could be construed as a potential conflict of interest.
Received: 01 August 2014; accepted: 01 October 2014; published online: 03 November 2014.

Citation: Mirabella G (2014) Should I stay or should I go? Conceptual underpinnings of goal-directed actions. Front. Syst. Neurosci. 8:206. doi: 10.3389/fnsys.2014.00206 This article was submitted to Frontiers in Systems Neuroscience.

Copyright (c) 2014 Mirabella. This is an open-access article distributed under the terms of the Creative Commons Attribution License (CC BY). The use, distribution or reproduction in other forums is permitted, provided the original author(s) or licensor are credited and that the original publication in this journal is cited, in accordance with accepted academic practice. No use, distribution or reproduction is permitted which does not comply with these terms. 